\title{
Self-Assembly Mechanism of pH-Responsive Glycolipids: Micelles, Fibers, Vesicles, and Bilayers
}

\author{
Niki Baccile, ${ }^{* \dagger}$ Anne-Sophie Cuvier, ${ }^{\dagger}$ Sylvain Prévost, ${ }^{\ddagger}$ Christian V. Stevens, ${ }^{\S}$ Elisabeth Delbeke, ${ }^{\S}$ \\ Jan Berton, ${ }^{,}$Wim Soetaert, "Inge N. A. Van Bogaert," and Sophie Roelants ${ }^{\|, \perp}$ \\ †Sorbonne Universités, UPMC Univ Paris 06, CNRS, Collège de France UMR 7574, Chimie de la Matière Condensée de Paris, UMR \\ 7574, F-75005 Paris, France \\ ${ }^{\ddagger}$ ESRF - The European Synchrotron, High Brilliance Beamline ID02, 38043 Grenoble, France \\ ${ }^{\S}$ SynBioC, Department of Sustainable Organic Chemistry and Technology, Ghent University, Ghent, Belgium \\ "InBio, Department of Biochemical and Microbial Technology, Faculty of Bioscience Engineering, Ghent University, Coupure Links \\ 653, 9000 Ghent, Belgium \\ ${ }^{\perp}$ Bio Base Europe Pilot Plant, Rodenhuizekaai 1, 9042 Ghent, Belgium
}

Supporting Information

ABSTRACT: A set of four structurally related glycolipids are described: two of them have one glucose unit connected to either stearic or oleic acid, and two other ones have a diglucose headgroup (sophorose) similarly connected to either stearic or oleic acid. The self-assembly properties of these compounds, poorly known, are important to know due to their use in various fields of application from cleaning to cosmetics to medical. At basic $\mathrm{pH}$, they all form mainly small micellar aggregates. At acidic $\mathrm{pH}$, the oleic and stearic derivatives of the monoglucose form, respectively, vesicles and bilayer, while the same derivatives of the sophorose headgroup form micelles and twisted ribbons. We use pH-resolved in situ small angle X-ray scattering (SAXS) under synchrotron radiation to characterize the $\mathrm{pH}$-dependent mechanism of evolution from micelles to the more complex aggregates at acidic $\mathrm{pH}$. By pointing out the importance of the $\mathrm{COO}^{-} / \mathrm{COOH}$ ratio, the melting temperature, $T_{\mathrm{m}}$, of the lipid moieties, hydration of the glycosidic headgroup, the packing parameter, membrane rigidity, and edge stabilization, we are now able to draw a precise picture of the full self-assembly mechanism. This work is a didactical illustration of the complexity of the self-assembly process of a stimuli-responsive amphiphile during which many concomitant parameters play a key role at different stages of the process.

\section{INTRODUCTION}

Stimuli-responsive self-assembly is an active field of research in the domain of soft matter due to the broad number of potential applications. ${ }^{1}$ Block copolymers and lipids are commonly designed to tune their self-assembly properties in solution as a function of temperature (e.g., use of $\mathrm{N}$-isopropylacrylamide residues), $\mathrm{pH}$ (use of amine or carboxylic acid residues), ionic force (use of charged residues), etc. ${ }^{2}$ Typical self-assembled structures range, for example, from simple spherical to branched micelles, disks, vesicles, sheets, and fibers, ${ }^{1,3-5}$ and the morphological relationship between them is generally considered to rely on the molecular structure, according to the well-known packing parameter relationship. ${ }^{6}$ Cone-like molecular morphologies tend to form curved objects, like micelles, while cylinder-shaped molecules rather form bilayers. Although this simplistic view can explain or even predict the shape of the micellar aggregates for various amphiphilic systems on a thermodynamic basis (e.g., ethylene oxide, ionic, gemini surfactants, ...), ${ }^{7-10}$ it fails to describe many experimental facts, as this is well-known among physico-chemists. ${ }^{11}$ Other

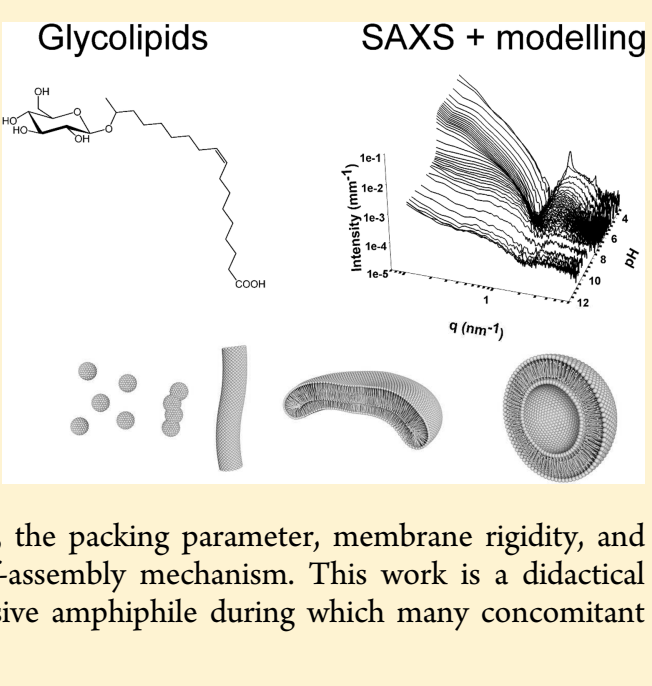

factors, often concomitant, play crucial roles in determining the structure-property relationship in soft systems. Typical factors influencing the self-assembly properties of lipids and block copolymers are the presence of Coulomb attraction/repulsion forces, the nature of counterions, temperature, hydration, rigidity of the aliphatic chain, entropy gain/loss, kinetics, steric repulsion, chirality, $\mathrm{H}$-bonding, mechanical, etc. The following examples of surfactants illustrate this point: gemini surfactants, and in particular quaternary ammonium-based ones, are known to form micelles in water. ${ }^{12}$ However, in the presence of an organic acid as a counterion, they can form helical/twisted fibers. ${ }^{13}$ Fatty acid salts are known to form micelles under basic $\mathrm{pH}$ conditions, whereas they become immiscible in their acidic form. However, in a narrow $\mathrm{pH}$ range between 7 and 9, they are able to form vesicular objects under accurate control of the starting conditions and the $\mathrm{COO}^{-} / \mathrm{COOH}$ ratio. ${ }^{5}$ Phospholi-

Received: June 23, 2016

Revised: September 27, 2016 
pids, the major constituent of the cell membrane, generally form planar bilayers. However, upon change of the water osmotic pressure or upon introduction of a cosurfactant (bile salts are often described in the literature), the bilayer membrane curvature can be modified. ${ }^{14}$ Vesicles, interesting objects for their encapsulation properties which are often used in drug delivery applications, ${ }^{1}$ are commonly obtained through mechanical treatment (shear force, filtration, sonication) of lamellar phospholipid phases. ${ }^{15}$ The examples above show the high degree of complexity in the fundamental understanding of amphiphilic systems and, above all, in the beforehand prediction of the most stable final structure. A further example is provided by the self-assembly properties of peptide nanotubes, for which the mechanism of formation was reported to depend on the way they are prepared, either by dispersion of the peptide in water at room temperature (dissolution reassembly mechanism) or by a heating-cooling step (oriented attachment). ${ }^{16}$ If, in this specific case, the process is believed to depend on the different supersaturation level of the peptide in solution, the importance of kinetics in self-assembly has also been emphasized in the past decade, as largely described by Leng et al.; ${ }^{17}$ for instance, the rate at which a given stimulus is applied (e.g., slow variation in temperature or in $\mathrm{pH}$ induces the formation of large, or more aggregated, vesicles in tetraethylene glycol-based or phospholipid amphiphiles) is known to have a potential effect. ${ }^{18,19}$ If an exhaustive list of examples is practically impossible, the main message is that it is impossible nowadays to predict the self-assembly properties in solution of a given compound on the sole basis of its molecular structure.

In this work, we present four microbial sugar-based bolaamphiphiles, of which the chemical structures are closely related and of which the self-assembly behavior could be expected to be similar. Two structurally similar sophorolipid molecules have been selected, both containing a glucose $\beta(1,2)$ disaccharide (sophorose) as the hydrophilic head, but a different C18 fatty acid tail, namely, oleic acid for the SLC18:1 (monounsaturation in position 9,10) compound and stearic acid for the SL-C18:0 (fully saturated), as shown in Figure 1. The second set of compounds has exactly the same fatty acid tails but a single $\beta$-D-glucose hydrophilic headgroup, instead of the disaccharide (G-C18:1 and G-C18:0 compounds, Figure 1).

Sugar-based amphiphiles are a class of molecules which attract much attention due to their biobased origin combined

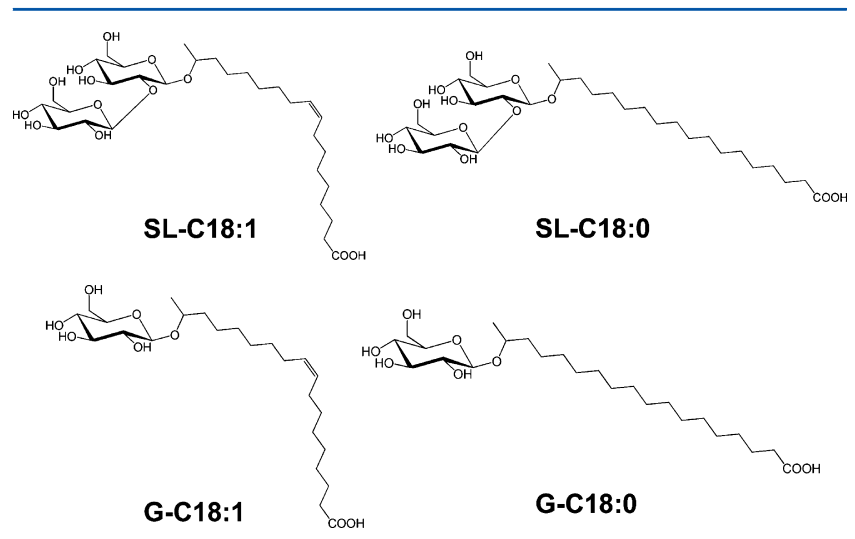

Figure 1. Chemical structures of SL-C18:1 and SL-C18:0 sophorolipids and G-C18:1 and G-C18:0 glucolipids. with their astonishing self-assembly properties in water. ${ }^{20-25}$ The broad variety of supramolecular structures (micelles, vesicles, fibers, bilayers, chiral fibers $)^{26-28}$ they can form makes them valuable substitutes for synthetic, more classical families of amphiphiles, like alkylammonium and alkylsulfate salts or block copolymers, yet these sugar-based amphiphiles exhibit a rich self-assembly behavior. Given the importance of carbohydrates in glycobiology and medicine, ${ }^{29,30}$ and the richness of carbohydrate chemistry, the applications of glycolipids are potentially broader than those for engineered amphiphiles. ${ }^{31}$ In addition, the biobased microbial origin of the glycolipids selected for this study makes them interesting alternative candidates to both petroleum-based compounds. Microbial glycolipids, also known as "biosurfactants", have a good biodegradability, low cytotoxicity, and numerous applications as antimicrobial, antibiofilm, anticancer, emulsifying, and stabilizing agents already exist. ${ }^{32-34}$ Scaled-up production is nowadays possible, as various companies (e.g., Evonik, Intobio, Jeneil, Soliance) have an ongoing biosurfactants (sophorolipids in particular) production. If counter arguments against biosurfactants state that the cost is still high due to limited production and purification processes, in the long run, this field is expected to grow more and more, as shown by the new opportunities offered by the selection of genetically modified strains ${ }^{35}$ to increase both productivity and selectivity.

The glycolipids selected for this work are characterized by two well-known chemical groups (glucose, fatty acid) which allow direct comparison with more common amphiphiles (phospholipids, anionic and neutral surfactants, fatty acid salts, alkyl polyglycosides, just to cite some). However, their bolaform structure, the unusual behavior of sugars (e.g., via oriented hydrogen bonding ${ }^{36,37}$ ), and $\mathrm{pH}$-responsive properties are factors which unpredictably influence the self-assembly. At basic $\mathrm{pH}$, all selected compounds form micelles as a major component, although the presence of additional aggregates (platelets, bilayers, ill-defined structures) must also be outlined. ${ }^{38,39}$ At acidic pH, SL-C18:1 and SL:C18:0 sophorolipids respectively assemble into micelles ${ }^{40,41}$ and twisted ribbons; ${ }^{42}$ in addition, G-C18:1 glucolipids form vesicles while G-C18:0 forms infinite bilayer sheets. ${ }^{39}$ The aim of this study is to find insights into the structure-property relationships and $\mathrm{pH}$ driven assembly mechanism for a set of structurally related microbial glycolipids using $\mathrm{pH}$-resolved in situ small angle X-ray scattering (SAXS). In particular, we will show the tight relationship between the $\mathrm{COOH} / \mathrm{COO}^{-}$ratio, the carbohydrate hydration, the lipid melting temperature, $T_{\mathrm{m}}$, membrane flexibility, and the domain of stability for micelles, ribbons, vesicles, and bilayers.

\section{MATERIALS AND METHODS}

Microbial glycolipids are known to be a mixture of similar congeners, of which one form is generally the majority ( $>85 \%)$. Figure 1 shows the chemical structures of the most abundant forms of sophorolipids SL-C18:1 and SL-C18:0 and glucolipids G-C18:1 and G-C18:0. SLC18:1 is obtained from the yeast Starmerella bombicola; its synthesis was described elsewhere, ${ }^{41}$ and the relative HPLC-ELSD data and composition are given in Figure S1. SL-C18:0 is obtained from the monounsaturated SL-C18:1 by a chemical modification step. ${ }^{42}$ Glucolipid G-C18:1 has been obtained using a modified strain ( $\Delta u g t B 1)$ of the yeast S. bombicola, while G-C18:0 is derived from GC18:1 through hydrogenation. Sophorolipids are characterized by a glucose $\beta(1,2)$ disaccharide hydrophilic headgroup, while glucolipids contain a single $\beta$-D-glucose hydrophilic headgroup. The synthesis 
Table 1. Main Components of the Sophorolipid (SL-) and Glucolipid (G-) Compounds Used in This Study ${ }^{a}$

\begin{tabular}{|c|c|c|c|c|c|c|c|}
\hline \multirow[b]{2}{*}{ sample } & \multicolumn{5}{|c|}{ congener } & \multicolumn{2}{|c|}{ references } \\
\hline & C18:1, subterminal & C18:1, terminal & C18:2, subterminal & C18:0, subterminal & C18:0, terminal & analysis by HPLC-ELSD & former studies \\
\hline SL-C18:1 & 86.0 & 6.0 & 1.0 & 1.0 & 0 & this work & 38,41 \\
\hline SL-C18:0 & 0 & 0 & 0 & 90 & 10 & 42 & $38,42,43$ \\
\hline G-C18:1 & 89.6 & 7.3 & 1.3 & 1.8 & 0 & 39 & 39 \\
\hline G-C18:0 & 0 & 0 & 0 & 92.7 & 7.3 & 39 & 39 \\
\hline
\end{tabular}

${ }^{a} \mathrm{C} 18: 2, \mathrm{C} 18: 1$, and $\mathrm{C} 18: 0$ refer to the number of carbons (C18) in the fatty acid chain and number of unsaturations $(2,1$, or 0$)$. Subterminal (C17) and terminal $(\mathrm{C} 18)$ refer to the position of the glycosidic bond in the fatty acid tail with respect to the COOH group (C1). The percentages refer to values estimated by measuring the peak area ratio in HPLC-ELSD measurements. Residual congeners in the SL-C18:1 sample batch are given in the Supporting Information (Figure S1). References indicate previous studies in which these same sample batches have been employed.

procedure and analysis of glucolipids is provided in ref 39. All C18:1 compounds contain a monounsaturation at position $\mathrm{C} 9,10$ of the fatty acid, while C18:0 compounds are characterized by a fully saturated chain.

Table 1 gives the exact composition of each sample used in this work determined by the peak area ratio in HPLC-ELSD measurements ${ }^{42,39}$ as well as the previous studies in which these same batches have been used. Upon use, all compounds are in their acidic $(\mathrm{COOH})$ form and fully deacetylated. The most abundant species (close to $90 \%$ on average) is composed by the glycolipid having the glycosidic bond in the subterminal, C17, position, as indicated in Figure 1. However, unavoidable amounts of congeners are always present, a common fact for all studies on biobased amphiphiles. The largest impurity common to all glycolipids is the terminal, C18, congener of each lipid and which systematically represents between 6 and $10 \%$ of the mixture for all compounds.

Sample Preparation. A given amount of the desired compound is dissolved into Milli-Q-grade water at room temperature to give a concentration of $5 \mathrm{mg} / \mathrm{mL}$. The $\mathrm{pH}$ is increased up to $\sim 11.6$ by adding $10-15 \mu \mathrm{L}$ of a $5 \mathrm{M} \mathrm{NaOH}$ solution, and it is eventually decreased by adding $\mu \mathrm{L}$ amounts of $0.1 \mathrm{M} \mathrm{HCl}$. This procedure, necessary to solubilize the samples, generates $\mathrm{NaCl}$. However, this is only a minor drawback because all systems are prepared in the same way and, consequently, comparable one to the other. In addition, it was shown that the effect of $\mathrm{NaCl}$ in these systems only influences aggregation: $\mathrm{NaCl}$ only affects the aspect ratio of SL-C18:1 micelles, ${ }^{41}$ while it impacts fiber aggregation, but not their chirality, on SL-C18:0 sophorolipids. ${ }^{43}$ Additional arguments supporting the low impact of $\mathrm{NaCl}$ will be discussed in the text.

pH-Resolved in situ Small Angle X-ray Scattering (SAXS). SAXS experiments have been done using a flow-through polycarbonate $2 \mathrm{~mm}$ capillary connected to the sample-containing solution at $\mathrm{pH}$ 11.6 through a peristaltic pump. The $\mathrm{pH}$ was controlled in situ via a classical $\mathrm{KCl} \mathrm{pH}$-meter directly located in the experimental hutch and monitored in real time. $\mathrm{pH}$ changes have been obtained by using a 0.1 $\mathrm{M} \mathrm{HCl}$ solution introduced via a motor-controlled press-syringe. If the continuous flow-through device avoids beam damage, we could only acquire one spectrum at each $\mathrm{pH}$ under this particular setup, thus making the statistics more fluctuating than classical ex situ experimental conditions. Data have been acquired on the high brilliance ID02 beamline at the ESRF synchrotron (Grenoble, France). $(E=12.46 \mathrm{keV}$, sample-to-detector distance $=1 \mathrm{~m})$ Error bars were calculated on the basis of the estimated number of photons detected (accounting for the gain and quantum efficiency of the CCD and phosphor layer), assuming Poisson statistics. The noise of the detector was accounted for by comparison of dark images. A CCD camera was used to collect the scattered photons and integrated azimuthally to obtain a typical $I(q)$ spectrum. Contribution of the solvent (water at $\mathrm{pH}$ 11.6) and capillary have been measured prior to the experiment and duly subtracted during the data treatment. All data have been corrected for the transmission of the direct beam and scaled to be in absolute scale. The detailed analysis of the SAXS data is presented in the Supporting Information (Figure S2).

Transmission Electron Microscopy (TEM). TEM experiments have been performed on an FEI Tecnai 120 Twin microscope operating at $120 \mathrm{kV}$ equipped with a Gatan Orius SC1000 CCD numeric camera $4 \mathrm{k} \times 4 \mathrm{k}$. A set of TEM images was recorded on a single copper TEM grid onto which a drop of SL-C18:0 solution at $\mathrm{pH}$ 11 and $5 \mathrm{mg} / \mathrm{mL}$ has been deposited and locally exposed to a concentrated $\mathrm{HCl}$ solution for a few seconds before the drop is dried. This operation allows one single grid to contain all objects formed at different stages of their growth, from the precursor micellar/platelet ${ }^{38}$ to the twisted ribbon ${ }^{42}$ state. Previous experiments ${ }^{43}$ have shown that, for this particular system, conventional TEM observation does not affect the fibrillar structure and morphology; for this reason, we use it here in a qualitative way to observe the intermediates of the twisted ribbon formation.

HPLC-ELSD Analysis. HPLC-ELSD analysis was performed with the Agilent 1260 Infinity equipped with an Agilent Zorbax Eclipse Plus C18 column $(4.6 \times 100 \mathrm{~mm}-3.5 \mu \mathrm{m})$ at $40^{\circ} \mathrm{C}$. A flow rate of $1 \mathrm{~mL} /$ min was applied, and a gradient of two solvents (A, 0.05\% acetic acid; $\mathrm{B}$, acetonitrile) was applied using the following method: $0 \mathrm{~min}, 95 \% \mathrm{~A}$ and $5 \% \mathrm{~B} ; 25 \mathrm{~min}, 5 \% \mathrm{~A}$ and $95 \% \mathrm{~B} ; 27 \mathrm{~min}, 5 \% \mathrm{~A}$ and $95 \% \mathrm{~B} ; 30$ $\min , 95 \% \mathrm{~A}$ and $5 \% \mathrm{~B}$.

LC-MS Analysis. LC-MS analysis of glucolipids was done on a Shimadzu LC-10-AD HPLC system (Shimadzu Europe GmbH, Germany) connected to a quadrupole mass spectrometer (Waters, Milford, MA). Molecules were identified by their native molecular masses after ESI (electron spray ionization) without collision.

\section{RESULTS AND DISCUSSION}

Basic $\mathrm{pH}$. The SAXS profiles and corresponding fits above $q$ $>1 \mathrm{~nm}^{-1}$ using a core-shell ellipsoid of revolution model for all microbial glycolipid samples at $\mathrm{pH} 11.0 \pm 0.3$ are given in Figure 2. As it was observed before, ${ }^{39}$ all samples have a similar scattering profile characterized by an intense signal below $q<$ $0.3 \mathrm{~nm}^{-1}$ and a signature typical for micellar objects above $q>1$ $\mathrm{nm}^{-1}$. Very interestingly, the first oscillation of the form factor above $2 \mathrm{~nm}^{-1}$ shows remarkable differences between all samples. From a qualitative point of view, SL-C18:1 and GC18:1 samples have a similar, rather flat, oscillation, which is, on the contrary, more pronounced for SL-C18:0 and G-C18:0 systems. It is well-known that specific ion binding on the hydrophilic polar headgroup modifies the electron spatial distribution with an obvious influence on the final form factor oscillation; ${ }^{44,45}$ however, in this situation, all samples are at $\mathrm{pH}$ 11 and therefore they contain a comparable amount of $\mathrm{Na}^{+}$. For these reasons, one must attribute the spectroscopic differences to a specific arrangement of the core-shell-solvent interfaces according to the type of glycolipid employed. To better understand that, we have fitted all curves with the same CoreShell Ellipsoid of Revolution model (CSERm) form factor (refer to the Supporting Information for more details) and we have proceeded as follows in order to understand which are the crucial parameters that have a direct impact on the form factor oscillation profile. 

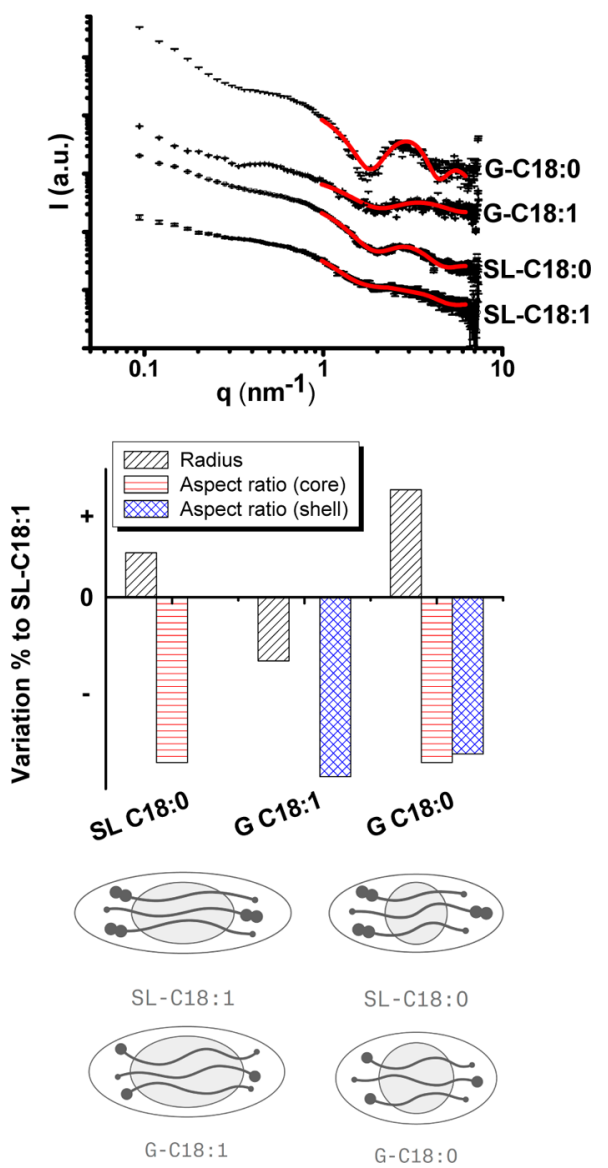

Figure 2. SAXS data (with error bars) corresponding to both sophorolipids and glycolipids $(0.5 \mathrm{wt} \%)$ at $\mathrm{pH} 11.0 \pm 0.3$. The red curves correspond to the fit performed using a core-shell ellipsoid of revolution form factor model. The fitting procedure for this system has been outlined in the Supporting Information. The histogram shows the relative variation of the most significant fitting parameters (radius, aspect ratio (core, shell)), keeping all others constant, for the SLC18:0, G-C18:1, and G-C18:0 samples and with respect to the reference compound, SL-C18:1. The modeled ellipsoid structures for each glycolipid are given on the bottom of the figure.

First of all, we operate a best fit procedure on the reference SL-C18:1 system, as this was largely studied before both at acidic and basic $\mathrm{pH} .{ }^{41,38}$ We use the values obtained in ref 38 as starting parameters. The optimized values for this specific system, reported in Table S1, are comparable to those reported in ref 38. We then use this set of values to fit the SL-C18:0 and G-C18:1 profiles, and we vary all parameters one-by-one so as to match the experimental curves at best. We find that the aspect ratio (core) has the strongest impact on the fit of the SLC18:0 sample, while the radius refines the result. We also find that simultaneous variation of shell SLD and aspect ratio (shell) is necessary to fit the G-C18:1 system, while changes of the radius, $R$, refine the result. Finally, we use the values found for the SL-C18:0 system to fit the G-C18:0 data and we observe that aspect ratio (shell) and radius only have a minor impact on the quality of the fit. Interestingly, the thickness, $T$, does not need variation in any of the systems and it was kept constant at $3.93 \times 10^{-1} \mathrm{~nm}$. We must stress the fact that these are not best fits but only adjustements to understand the least number of critical parameters accounting for the spectral differences above $q>1 \mathrm{~nm}^{-1}$. For this reason, the absolute values given in Table S1 should be taken with caution; however, the relative variations of the radius, aspect ratio (core), and aspect ratio (shell) in all samples with respect to SL-C18:1 are very informative, as shown in Figure 2.

Given an approximate micellar morphology for the SL-C18:1 sample at $\mathrm{pH} \sim 11$, depicted in Table S1, one can see that the saturation of the $\mathrm{C}_{9,10}$ double bond mainly impacts the micellar core, which becomes practically spherical, while the shell aspect ratio is unchanged (sample SL-C18:0). When keeping the unsaturation but reducing the number of sugar units (sample G-C18:1), the core aspect ratio is unchanged but the shell aspect ratio is reduced, as illustrated in Table S1. Finally, one glucose unit combined with saturation (sample G-C18:0) has a spherical core with a reduced shell aspect ratio.

These data can be generalized as follows: (1) The monounsaturation always drives the formation of an elliptical hydrophobic core, while saturated compounds always have a spherical core. (2) The higher the number of sugar units, the larger the shell size in the axial direction, at constant shell thickness in the equatorial direction. If the second observation is in agreement with the larger size of the sophorose headgroup if compared to glucose, both observations taken together could only be explained by the fact that in all samples the carbohydrate headgroups are mainly located in the axial direction of the shell while the carboxylate groups are probably located in both axial and equatorial directions of the shell. Meanwhile, all saturated (C18:0) compounds seem to form a homonegeous spherical core, while monounsaturated ones (C18:1) tend to be in an ellipsoidal volume, the difference between them probably originating from a combination between electrostatic repulsions of the carboxylate groups and differences in the rigidity of the aliphatic chain at room temperature. In all cases, the core size below $1 \mathrm{~nm}$ is in agreement with previous studies ${ }^{41}$ on sophorolipids, suggesting interpenetration and bending phenomena of the aliphatic chain, as predicted by Nagarajan ${ }^{46}$ on bolaform amphiphiles. Nevertheless, we are never able to describe the system with a homogeneous hydrophilic shell, a fact which, even if it was proposed by Nagarajan, ${ }^{46}$ was demonstrated not to be true in the case of SL-C18:1 sophorolipid micelles at $\mathrm{pH}$ below 7.41.

pH-Resolved Morphology Transitions. Addition of $\mathrm{HCl}$ to the systems described just above drives important morphological changes below a critical $\mathrm{pH}$ value, characteristic for each glycolipid. ${ }^{39}$ Turbidity experiments have shown that $\mathrm{pH}$ values of 7.4, 6.2, and 7.8 mark the transition for, respectively, SL-C18:0, G-C18:1, and G-C18:0 into twisted ribbons, vesicles, and infinite bilayer sheets. ${ }^{39,42}$ As for SLC18:1, no transition $\mathrm{pH}$ could be defined on the basis of light scattering experiments. ${ }^{42}$ The corresponding $\mathrm{pH}$-dependent in situ SAXS experiments on all samples are shown in Figure 3, while the corresponding contour plots are presented in Figure S3. From a qualitative point of view, one can see that all systems present a continuous spectral evolution from basic to acidic $\mathrm{pH}$ and that the spectral signature is unchanged from $\mathrm{pH}$ $\sim 12$ to $\mathrm{pH} \sim 8$. More specifically:

(a) The broad signal above $q>1 \mathrm{~nm}^{-1}$ for the SL-C18:1 system (Figure 3 ) becomes more visible at lower $\mathrm{pH}$, thus putting in evidence a clear rearrangement of the electron density distribution in the micelle, the characteristic signal of which ${ }^{41}$ also becomes evident in the low- $q$ region below $q<1 \mathrm{~nm}^{-1}$. The SL-C18:1 contour plot in Figure S3 shows the appearance of the first minimum of 

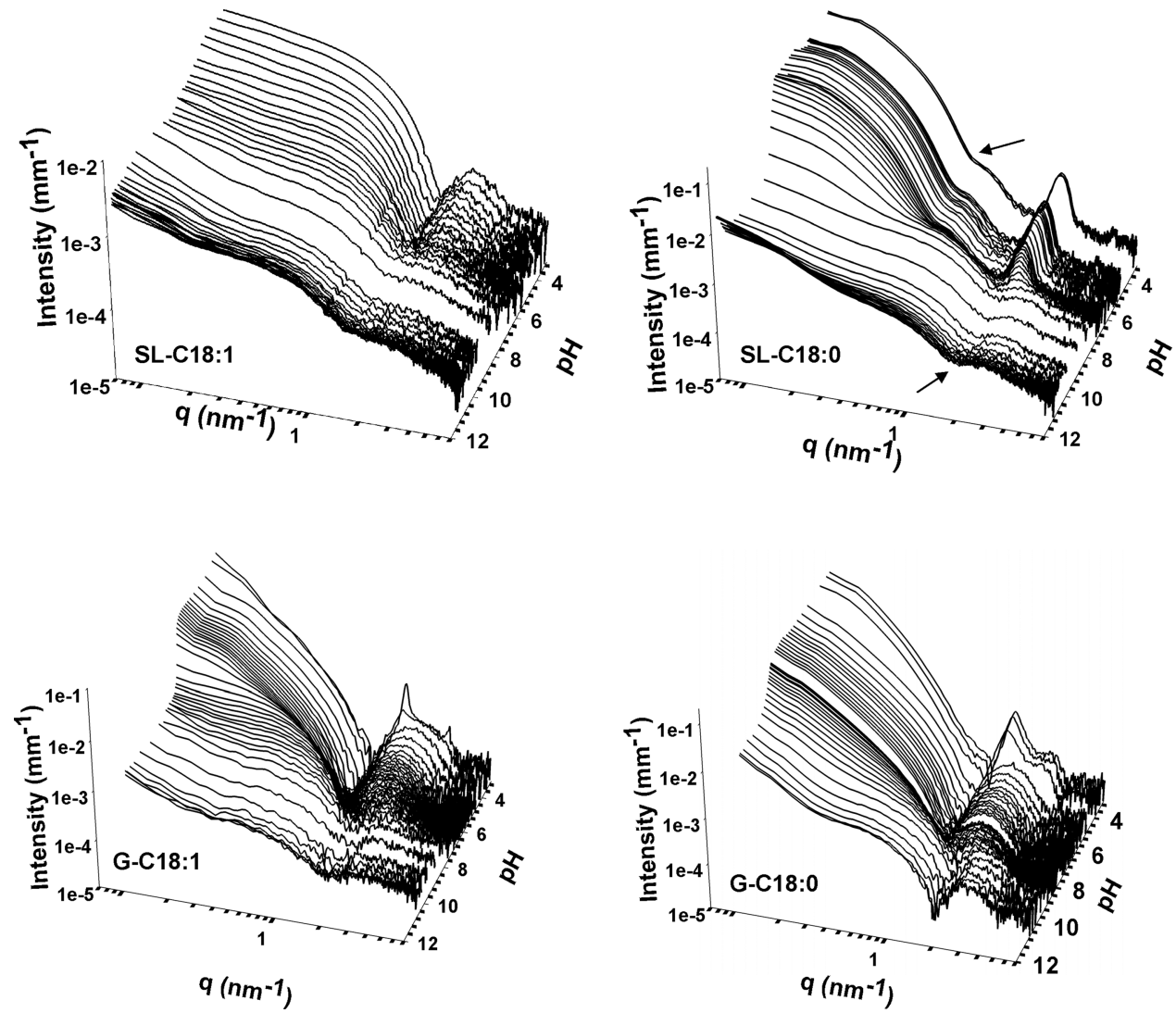

Figure 3. 3D line intensity plots of the pH-resolved SAXS experiments for all glycolipids at 0.5 wt $\%$ in water.

the form factor (white arrow) and intense scattering below $q<1 \mathrm{~nm}^{-1}$, at $\mathrm{pH}=\sim 6.5$.

(b) The G-C18:1 system in Figure 3 shows the evolution from the micellar region at $\mathrm{pH}$ above 8 to the vesicle domain $^{39}$ at acidic $\mathrm{pH}$, characterized by intense scattering at $q<1 \mathrm{~nm}^{-1}$ and the formation of a broad oscillation at $q>1 \mathrm{~nm}^{-1}$. Below $\mathrm{pH} 4$, a white precipitate forms, characterized by a diffraction pattern typical of a lamellar phase. ${ }^{39}$ Countour plots of G-C18:1 in Figure S3 visually show the phase transition in the $7.5<\mathrm{pH}<6$ region between the micelles and the vesicles by following the minimum of the form factor (arrow 1) and the appearance of the broad oscillation, attributable to the forming vesicles (arrow 2).

(c) The G-C18:0 system in Figure 3 evolves from a micellar system at basic $\mathrm{pH}$ toward a bilayer sheet at acidic $\mathrm{pH}^{39}$ As for the G-C18:1, the form factor oscillation at $q>1$ $\mathrm{nm}^{-1}$ (micelle) transforms into a broad oscillation attributable to a bilayer morphology. This is shown in the G-C18:0 contour plot in Figure S3, where one can see that the micelle-to-bilayer transition region is concentrated in a narrow $\mathrm{pH}$ range below 8.5.

(d) Finally, the SL-C18:0 system shown in Figure 3 shows a persistent micellar signature until a sharp transition to the twisted ribbon phase occurs, characterized by the appearance of a broad diffraction peak, characteristic of the molecular packing of these molecules within the ribbon plane. ${ }^{42}$ The contour plot in Figure S3 shows a direct view of the micelle-to-ribbon transition, the $\mathrm{pH}$ of which is situated in the vicinity of $\mathrm{pH}$ 7. The ribbon phase is stable down to $\mathrm{pH} 3$, at least.
To better understand which structural parameter drives the morphological changes between the phases at basic and acidic $\mathrm{pH}$, one can plot two model-independent parameters, the fractal dimension, $D_{f}$, and the minimum of the form factor oscillation, $q_{\min }$, as well as the model-dependent parameters. The $\mathrm{pH}$-dependent evolution of the micellar fit parameters in the region above $q>1 \mathrm{~nm}^{-1}$ (Figure 5) can also be used to understand their role in the transitions.

Large-Scale Objects and Evolution of $\boldsymbol{q}_{\mathrm{min}}$. The $\mathrm{pH}$ dependent evolution of the slope at $q$-values below $q<\sim 0.7$ $\mathrm{nm}^{-1}$ is informative at the morphological transitions on large scales $(>\sim 10 \mathrm{~nm})$. The value of the slope in log-log scale can either be related to a well-defined morphology (e.g., -1 for cylinders, -2 for flat objects $)^{47}$ or to fractal objects, ${ }^{48}$ even if polydispersity can also influence the value of the slope. Considering our data, we have analyzed the $q$-region below $q$ $<\sim 0.3 \mathrm{~nm}^{-1}$ and between $\sim 0.3<q\left(\mathrm{~nm}^{-1}\right)<\sim 0.7$ separately, where these values are to be considered as approximate, the exact ones being indicated in Figure 4. The SL-C18:1 sophorolipid (Figure 4) shows the presence of large scale objects (black line) coexisting with small micelles from basic $\mathrm{pH}$ down to $\mathrm{pH} \sim 7$, below which the size of the micelles increases to form cylinders (red line, -1 slope). This result agrees well with published data on this system, where nanoscale platelets coexist with micelles at $\mathrm{pH} 11,{ }^{38}$ while elongated ellipsoids form below $\mathrm{pH}$ 7. The data in Figure 4 show that the platelet aggregates are stable up to the $\mathrm{pH} \sim 8$ point at which elongated micelles are predominant. The concomitant increase in the micellar size (taken here as the radius of gyration) and the change of the oscillation profile of the form factor (refer to the white arrow in SL-C18:1 in Figure S3) suggest that the platelets may act as a reservoir for sophorolipid molecules. 

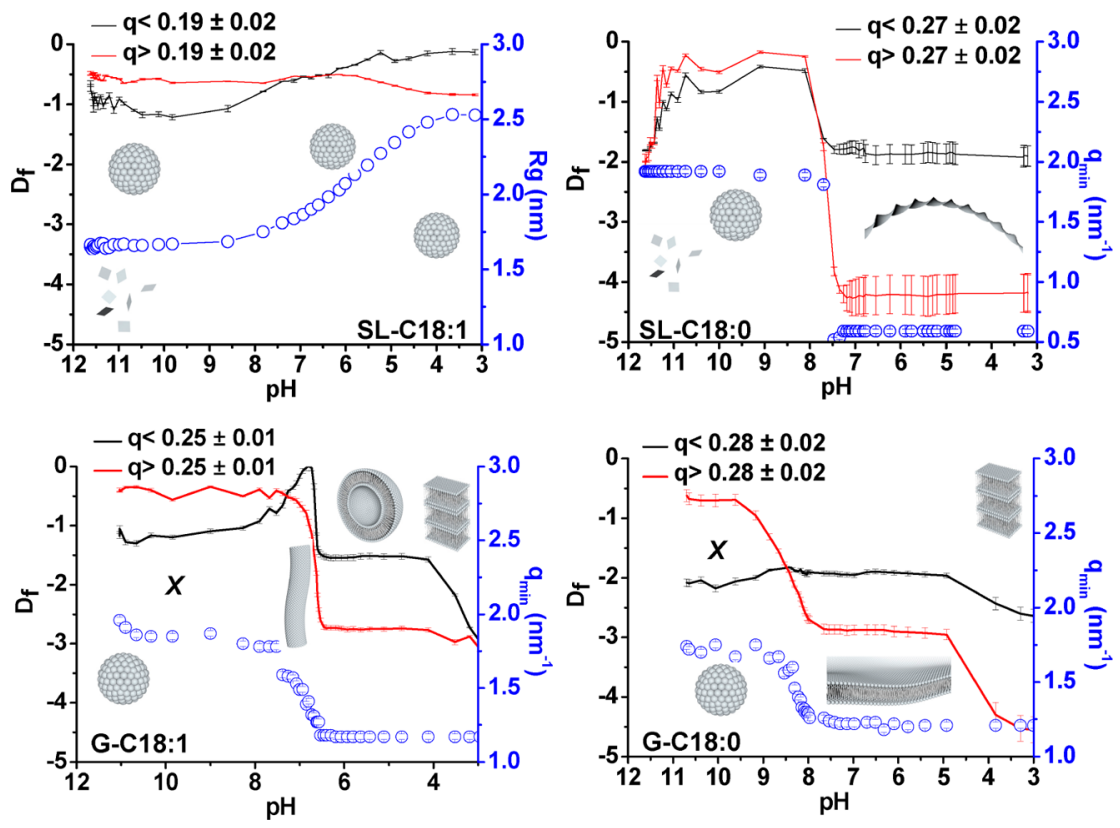

Figure 4. On the left-hand ordinates of each plot is reported the pH-dependent evolution of the fractal dimension, $D_{\mathrm{f}}$, estimated above and below a critical $q$-value indicated in each plot and different for each compound. On the right-hand ordinate of SL-C18:1, Rg (radius of gyration) is plotted against $\mathrm{pH}$. For the G-C18:1, G-C18:0, and SL-C18:0 systems, $\mathrm{Rg}$ cannot be determined straightforwardly; the minimum of the form factor oscillation, $q_{\min }$ (see Figure S2 for more details), as a function of $\mathrm{pH}$ is reported, instead, on the right-hand ordinates. " $X$ " refers to the concomitant presence of giant polymorphic structures with micelles in the basic $\mathrm{pH}$ region of G-C18:1 and G-C18:0 samples. ${ }^{39}$

The $\mathrm{pH}$-dependent behavior of the monoglucose G-C18:1 drastically changes. At basic $\mathrm{pH}$, the system is formed by micelles and some polymorph giant structures. ${ }^{39}$ The slope at low $q$ then gently decreases to zero at $\mathrm{pH} 7$, while a sharp transition takes place below $\mathrm{pH}$ 7. Interestingly, $q_{\min }$ decreases from about 1.85 to $1.16 \mathrm{~nm}^{-1}$ between $\mathrm{pH} 7$ and 8 . Remarkably, the $q_{\min }$ ratio before and after the transition point is about 1.59 , which is close to the theoretical value of 1.43 expected for a transition between full spherical to lamellar objects $(q R=4.49$ for a sphere and $\pi$ for a lamellae $)$, if no model assumption is made. Cryo-TEM data show that the micelles fuse together to form giant branched cylindrical micelles in the narrow $7.5<\mathrm{pH}<6.5$ region, as also demonstrated by the $D_{\mathrm{f}}$ value close to -1 in this $\mathrm{pH}$ range (Figure 4). After reaching a critical concentration, they then merge into vesicles below $\mathrm{pH} 6.6,{ }^{39}$ in full consistency with the $q_{\text {min }}$ evolution, and the close to -2 value for $D_{\mathrm{f}}$ in the low- $q$ region. This mechanism is further supported by the fact that the transition in $q_{\min }$ occurs during the $D_{\mathrm{f}}$ evolution toward -1 and before its stabilization at about -2 , thus showing that micelles merge together into cylinders before the morphological evolution from cylinders to vesicles. Finally, the vesicular system is very stable up to $\mathrm{pH} \mathrm{4}$, below which a white lamellar precipitate forms, as indicated by the presence of diffraction peaks in Figure 3. ${ }^{39}$

Upon suppression of the double bond, one forms the G$\mathrm{C} 18: 0$ compound, the $\mathrm{pH}$-dependent behavior of which is also shown in Figure 4. At basic $\mathrm{pH}$, this system contains both micelles and giant polymorph structures with a marked -2 slope (black curve), indicating their flat morphology. Interestingly, these objects are very stable and they increase in size and amount upon lowering the $\mathrm{pH}$. This is shown by the constant $D_{\mathrm{f}}=-2$ value below $q<0.28 \mathrm{~nm}^{-1}$ throughout the $\mathrm{pH}$ range, by the slight decrease in $q_{\min }$ from about 1.72 to 1.22 $\mathrm{nm}^{-1}$ below $\mathrm{pH} 8$, the ratio of which, 1.41 , is in very good agreement with the theoretical 1.43 expected for a sphere-tolamellae transition. Additional proof for a micelle-to-bilayer transition is given by the increase in $D_{\mathrm{f}}$ above $q>0.28 \mathrm{~nm}^{-1}$ between $\mathrm{pH} 9.5$ and 8 . Differently from G-C18:1, the fact that the increase in $D_{\mathrm{f}}$ occurs at the same time as the $q_{\text {min }}$ transition suggests that micelles coalesce and merge together into the infinite, pre-existing, bilayer membrane, without forming intermediate structures. Another hypothesis could also be that micelles act as an external reservoir of matter. The sheets are very stable until $\mathrm{pH} 4$, below which a white lamellar precipitate forms, as indicated by the presence of diffraction peaks in Figure 3 and as discussed elsewhere. ${ }^{39}$

The SL-C18:0 system in Figure 4 shows a hybrid behavior with respect to the other glycolipids. At basic $\mathrm{pH}$, micelles coexist with platelets. ${ }^{39}$ Interestingly, the platelets are less stable toward a decrease in $\mathrm{pH}$ than in SL-C18:1, as shown by the fast decreasing low- $q$ signal at $\mathrm{pH}<10$. Upon approaching the transition $\mathrm{pH}$ for this system at $\mathrm{pH} \sim 7.4$, the slope undergoes a sharp increase with the simultaneous appearance of the diffraction peak (Figure 3) indicating the formation of twisted ribbons. ${ }^{42} q_{\min }$ undergoes a large shift from 1.92 to $0.60 \mathrm{~nm}^{-1}$ (better highlighted by the arrows in Figure 3) respectively before and after ribbon formation. The latter value corresponds to an indicative size of about $10 \mathrm{~nm}$, a value consistent with the ribbon cross section of about $12-15 \mathrm{~nm}$ measured by cryoTEM. $^{42}$ Given the sharp transition between the micelles and the ribbons, one can make the hypothesis that micelles act more as a reservoir of matter for the ribbon than as a real precursor, a hypothesis which will be commented upon later.

pH-Dependent Evolution of Fit Parameters. To go further, we have performed a $\mathrm{pH}$-dependent simulation of the SAXS data using the simple core-shell ellipsoid of revolution model, useful to interpret the structural changes of the micelles with decreasing $\mathrm{pH}$. In Figure 5, we report the shell scattering length density (SLD), $\rho_{s}$, the equatorial core radius, $R$, the 

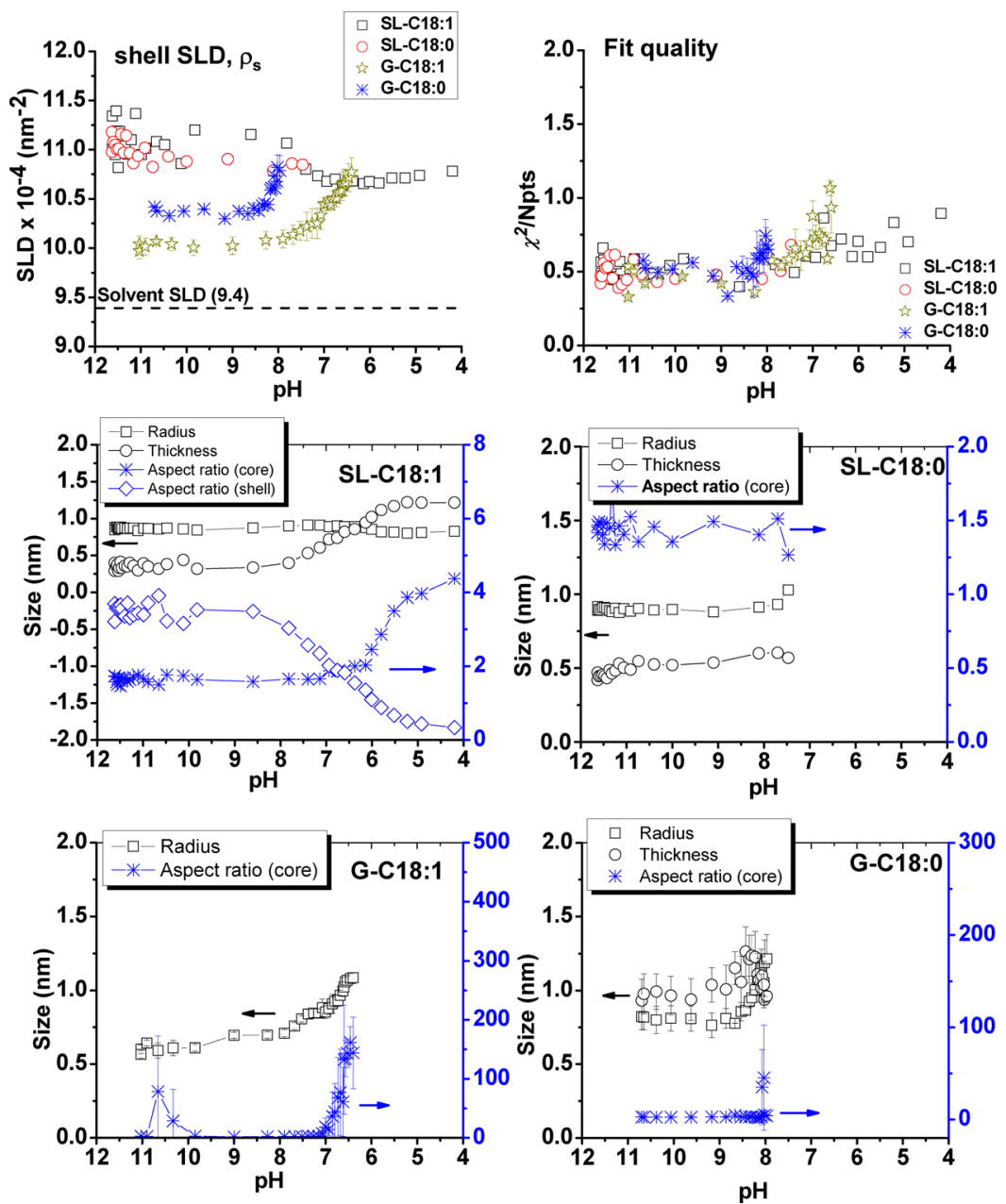

Figure 5. $\mathrm{pH}$-dependent evolution of the core-shell ellipsoid of revolution model fit parameters. SLD, evolution of the fitted shell scattering length density; fit quality, evolution of the $\chi^{2}$ /Npts quality control parameter. SL-C18:1, SL-C18:0, G-C18:1, G-C18:0 panels: evolution of the core radius, shell thickness (where applicable), and core and shell (where applicable) aspect ratios for the core-shell ellipsoid of revolution form factor model (the details of the model are given in the Supporting Information).

equatorial shell thickness, $T$, and core and shell aspect ratio along with the $\chi^{2} /$ Npts.

When looking at the data evolution with $\mathrm{pH}$ for the SLC18:1 sophorolipid, one can observe a stable radius and an increasing thickness; interestingly, upon approaching the transition $\mathrm{pH} \mathrm{7,} \mathrm{the} \mathrm{core} \mathrm{aspect} \mathrm{ratio} \mathrm{is} \mathrm{strongly} \mathrm{enhanced,}$ indicating the formation of elongated micelles, while the shell aspect ratio shrinks to zero. This result is in agreement with previous data collected on salt-containing SL-C18:0 sophorolipids in water at $\mathrm{pH}$ below 7 under static conditions. ${ }^{41}$ The same sample has been used here and in ref 41 . This set of data shows a local molecular redistribution within the micelle itself upon decreasing the $\mathrm{pH}$, that is upon increasing the amount of $\mathrm{COOH}$ groups; decreasing the amount of $\mathrm{COO}^{-}$groups consequently reduces electrostatic repulsion between the charged carboxylate, and one expects a reduction of the micellar local curvature, the effect of which is a local redistribution of sophorose from the axial to the equatorial region. The intrinsic value of the packing parameter $(p=0.36)$ of SL-C18:1 $1^{39}$ then seems to drive the formation of the elongated micelles at low $\mathrm{pH}$.

The $\mathrm{pH}$-dependent data of glucolipid G-C18:1 shows a 2fold increase in the radius and an important increase in core aspect ratio, meaning an increase in the ellipsoid length. This morphological change is in agreement with the formation of giant cylindrical micelles, outlined earlier on the basis of a $D_{\mathrm{f}}$ analysis, and previously shown by means of cryo-TEM data. This continuous evolution of spherical toward cylindrical micelles and eventually vesicles shows the tight structural relationship between the micelles at basic $\mathrm{pH}$ and the vesicles at acidic $\mathrm{pH}$.

The saturated glucolipid G-C18:0 shows similar features in terms of radius and thickness evolution, but it also shows that the core aspect ratio is constant throughout the $\mathrm{pH}$ range, thus confirming the strong stability of the micelles down to $\mathrm{pH} 8$. This also confirms the previous hypothesis according to which a direct transition from micelles to bilayer membranes occurs without intermediates, probably via a direct micellar fusion or a transfer of amphiphile. Similarly, the $\mathrm{pH}$-independent stability of the micellar structural parameters (Figure 5) for the saturated sophorolipid SL-C18:0 until the formation of the twisted ribbon at $\mathrm{pH} \sim 7.4$ confirms the hypothesis that in this system micelles play a minor role in the ribbon formation, probably acting as a reservoir of matter. Interestingly, the micelles composed of both saturated compounds, G-C18:0 and SL-C18:0, display the strongest structural stability toward $\mathrm{pH}$ changes, if compared to the monounsaturated compounds. 

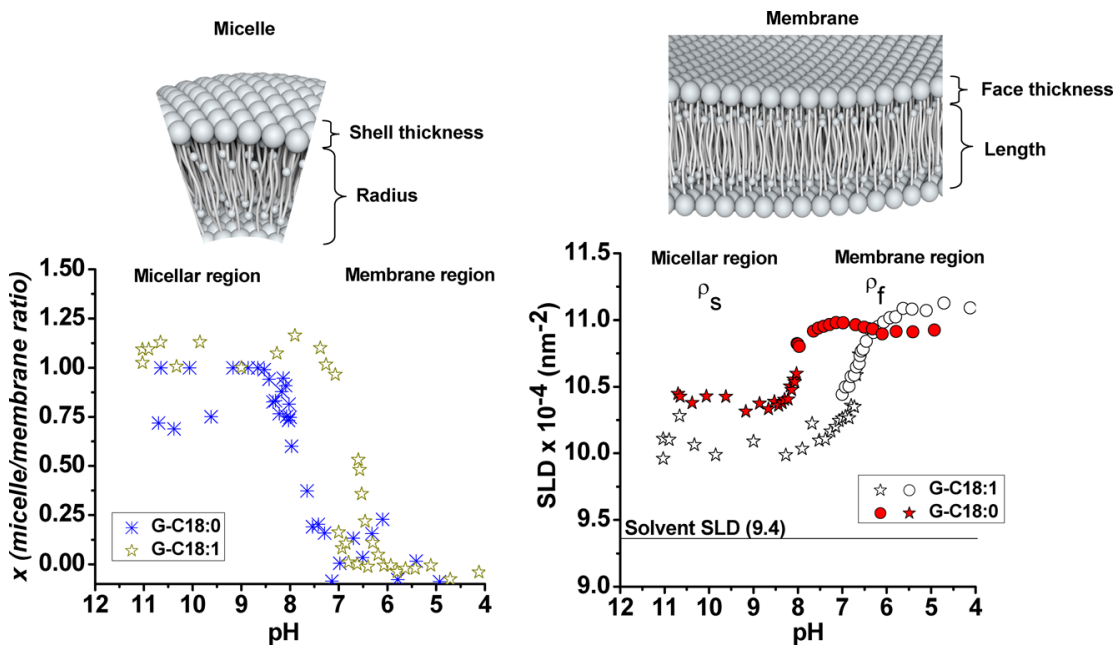

Figure 6. Left-hand panel: pH-dependent evolution of the micelle-to-membrane ratio, $x$, evaluated using eq S3 and the methodology of which has been given in the Supporting Information. Right-hand panel: $\mathrm{pH}$ evolution of the shell SLD, $\rho_{\mathrm{s}}$, and face SLD, $\rho_{\mathrm{f}}$, for the G-C18:1 and G-C18:0 glucolipids $(0.5 \mathrm{wt} \%) . \rho_{\mathrm{s}}$ refers to the SLD of a core-shell ellipsoid form factor used to model the data in the micellar stability region and identified by the star symbols. Core radius and shell thickness are additional free variables. $\rho_{\mathrm{f}}$ refers to the SLD of a core-shell bicelle form factor used to model the data in the bilayer membrane stability region and identified by the circle symbols. Face thickness and membrane length have also been set free in the fitting process. More details on the fitting procedure are given in the Supporting Information.

Finally, the most interesting feature for all systems, discussed in detail later, is probably the $\mathrm{pH}$-dependent evolution of $\rho_{\mathrm{s}}$ (Figure 5). In the case of sophorolipids, the starting $\rho_{\mathrm{s}}$ values are comparable for both SL-C18:1 and SL-C18:0 samples and higher than those for the glucolipids. In addition, $\rho_{\mathrm{s}}$ for sophorolipids seems to decrease with decreasing $\mathrm{pH}$, while it clearly increases for glucolipids in the vicinity of the corresponding transition $\mathrm{pH}$ values.

The drawback of the data in Figure 5 is the limited validity of the ellipsoid model in the vicinity of the transition $\mathrm{pH}$ for all systems except SL-C18:1. To confirm the $\rho_{\text {s }}$ trends, we have also modeled the bilayer region below the transition $\mathrm{pH}$. Figure 6 shows the $\mathrm{pH}$-driven evolution of the micelle/membrane ratio ( $x$, in eq S3) between unity (micelles only) and zero (membrane only) with decreasing $\mathrm{pH}$, nicely demonstrating the quantitative morphological evolution from micelles to bilayer membranes (vesicles or sheets). As for the corresponding SLD, the $\mathrm{pH}$-dependent evolution of $\rho_{\mathrm{s}}$ (the shell SLD in the ellipsoids) and $\rho_{\mathrm{f}}$ (the face SLD in the bilayer) combined in a single set of data before and after the transition $\mathrm{pH}$ value is also plotted in Figure 6. These data confirm and actually complete the evolution presented in Figure 5: the $\mathrm{pH}$-dependent increase of the SLD in the hydrophilic region for the glucolipid compounds.

SLD of the hydrophilic region (shell or face) is strongly sensitive to hydration in both the ellipsoid and bicelle models: upon hydration, the value of SLD decreases, while upon dehydration, SLD increases. According to the data presented in Figure 5 and Figure 6, it seems that the hydrophilic shell/water palisade undergoes dehydration in both glucolipids when $\mathrm{pH}$ decreases, while the opposite occurs in the presence of sophorose. We believe that, under basic $\mathrm{pH}$ conditions, the presence of the carboxylate group, besides influencing the local curvature due to electrostatic repulsion, also contributes to the hydrophilicity of the carbohydrate shell in all compounds, also due to the presence of the sodium counterion, which brings between 4 and 8 water molecules; ${ }^{49}$ monosaccharides like glucose are known to have a small hydration number, less than $3,^{50,51}$ while this value was estimated to be above 18 for sophorolipids in acidic $\mathrm{pH}$ medium in the presence of $\mathrm{RbCl}^{41}$ These assumptions indicate that, upon lowering the $\mathrm{pH}$ and subsequent formation of $\mathrm{COOH}$ groups, the contribution of sodium to carbohydrate hydration becomes less important, and under these conditions, one can reasonably expect the natural hydration layer of the sugar to become the main source of structural water, which is higher for sophorose than glucose, as it results from the SLD trends. In $\mathrm{pH}$-responsive lipidic systems, similar arguments have been specifically used to explain lamellar (low curvature) to hexagonal (high curvature) phases. $\mathrm{Li}$ et al. ${ }^{52}$ have developed a model for anionic lipids mixed either with neutral or cationic lipids showing that the $\mathrm{pH}$-dependent instability of vesicles is highly dependent on the membrane composition and in particular on the flow of counterions and their hydration shell. When these move away from the surface, the bilayer curvature decreases because the effective surface area of the lipid decreases. When hydrated counterions approach the surface, on the contrary, higher curvatures are stabilized. Their model fits well both previous experimental results by $\mathrm{Hafez}$ et al. ${ }^{53,54}$ and our own data, which can be considered to come from a mixture of an anionic $\left(\mathrm{COO}^{-} \mathrm{Na}^{+}\right)$and neutral $(\mathrm{COOH})$ lipid mixture, of which the composition continuously evolves with $\mathrm{pH}$. The mechanical deformation of lipid membranes due to water addition/removal has been known for a long time under the name of "osmotic stress", first described by LeNeveu ${ }^{55}$ in 1977 for lecithin bilayer and later exploited by many other authors. ${ }^{14,56,57}$ In particular, Rand et al. ${ }^{14}$ have shown that a variation of osmotic pressure is also able to modify the curvature radius of a lipid bilayer from lamellar to hexagonal, thus explaining from a fundamental point of view Li's ${ }^{52}$ model and, most likely, our own data.

In view of the discussion above, we then believe that the stronger water-withdrawing effect induced by $\mathrm{pH}$ in the glucose-based compounds contributes, at the same time as the reducing inter- $\mathrm{COO}^{-}$electrostatic repulsion and $\mathrm{Na}^{+}$ removal, to decrease the effective area per headgroup. Under these conditions, the hydrophilic headgroups come closer together. According to the simple packing parameter model, one can explain the formation of objects with a lower curvature, 
as this is the case for the lipid membranes with respect to the starting micelles. The calculated packing parameter for G-C18:1 is about $0.72,{ }^{39}$ which, combined with an additional waterwithdrawing effect occurring during lowering the $\mathrm{pH}$, can largely explain the micelle-to-cylinder-to-vesicle transition observed for this compound. However, the sudden bilayer formation and the strong stability of the micellar regime observed for G-C18:0 cannot be explained using these arguments. The different behavior in the structural evolution in the vicinity of the transition $\mathrm{pH}$ between these two systems is also clear from the plot of the micelle and membrane structural parameters (radius vs shell thickness and length vs face thickness), the details of which are given in the Supporting Information. Figure S4 shows a nice, continuous overlap between the micellar radius $(\sim 0.6 \mathrm{~nm})$ and shell thickness $(\sim 0.8 \mathrm{~nm})$ with the bilayer membrane length $(\sim 1 \mathrm{~nm})$ and respective face thickness $(\sim 1 \mathrm{~nm})$ with $\mathrm{pH}$ for the G-C18:1 sample. On the contrary, a clear structural evolution inconsistency exists between the micellar radius and shell thickness with respect to the bilayer membrane length and face thickness for the G-C18:0 sample. Both samples can be described by an interdigitated bilayer, because the its size (about $3 \mathrm{~nm}$ ) is comparable with the typical molecular dimensions, as discussed in the Supporting Information.

Formation of Bilayer Structures. G-C18:1 and G-C18:0 samples share a similar local bilayer structure, although the former assembles into a vesicle while the latter into an infinite bilayer. The assemblying pathway is also different. Micelles composed of G-C18:1 fuse into wormlike objects which in turn merge into disks; on the contrary, micelles composed of GC18:0 are stable toward $\mathrm{pH}$ and serve as a reservoir for the infinite growth of a pre-existing flat structure. If a common point is the presence of a flat bilayer at a given $\mathrm{pH}$, characteristic for each molecule, what is the driving force that induces the disk bending in the G-C18:1 case and disk growth for G-C18:0? We should recall that this behavior is valid at room temperature, that is, above and below the oleic acid and stearic acid melting temperatures, respectively $\left(13{ }^{\circ} \mathrm{C}\right.$ for oleic and $69{ }^{\circ} \mathrm{C}$ for stearic acid), the fatty acid components of, respectively, G-C18:1 and G-C18:0. ${ }^{39}$

To attempt to explain the $\mathrm{pH}$-dependent behavior of these two compounds at room temperature, one could refer to the work by Leng et al., ${ }^{17}$ who elegantly explained the micelle-tovesicle transition mechanism in a lecithin-bile salt binary system. They have shown how this is overall governed by kinetics but, at a local scale, by the lecithin (neutral)/bile salt (charged) ratio and salt concentration. They discuss the role of bile salt as an "edge-actant", an edge stabilizing agent, ${ }^{58}$ responsible for the stability of the bilayer membrane toward growth and, eventually, closure into vesicles. Bile salt preferentially sits at and stabilizes the outer edges of the lecithin membrane, contributing to reduce the local line tension, caused by the exposure of the hydrophobic chain of lecithin to the aqueous environment. The interplay between the lecithin-rich membrane disk growth and increase in line tension governs the instability of the disk at its edge and consequently the closure into a vesicle. Authors define a vesiculation index, $V_{\mathrm{f}}$, which is proportional to the membrane radius and line energy and inversely proportional to the bending energy of the membrane. For $V_{\mathrm{f}}<1$, flat membranes are stable, but for $V_{\mathrm{f}}>2$, vesicles become stable. In between, both membranes and vesicles are stable. Qualitatively, vesicles form whenever the bending energy of the membrane is small, that is, for elastic, flexible, objects, and upon growth (large membranes are easier to bend). To this logic phenomenon, one should add the contribution of line tension; at high values of line tension, $V_{\mathrm{f}}$ increases and vesicles form, occurring for those systems in which no edge rim stabilizes the membrane: vesicle formation is then preferred toward a stable membrane. If, for a given compound, the bending energy is constant while the membrane radius evolves with time, the edge stability will depend on the area fraction of edge-actant in the rim (which will in turn depend on the volume fraction of the edge-actant in solution) and salt concentration. Large amounts of edge-actants in the rim stabilize the rim itself by exposing the charged hydrophilic headgroups toward the solvent, instead of the lecithin hydrocarbon chains, while large amounts of salt screen the electrostatic repulsions between the edge-actant (charged) headgroups, thus contributing even more to membrane edge stability. Leng et al. could show that for large amounts of area fraction occupied by bile salt (the edge-actant) in a lecithincomposed membrane, and for a given salt concentration, the membrane becomes highly stable even at radii as large as 200 $\mathrm{nm}$ (very small $V_{\mathrm{f}}$ values). The higher the salt in solution, the more stable the membrane.

If we apply the main conclusions of the above study to the glucolipid systems presented here, provided certain hypotheses developed in the Supporting Information, we find one strong analogy: the $\mathrm{pH}$ variation practically imposes a continuous evolution in the $\mathrm{COOH}$ (neutral) $/ \mathrm{COO}^{-} \mathrm{Na}^{+}$(charged) proportion in solution, a parameter which is analogous to the lecithin (neutral)/bile salt (charged) ratio described by Leng et al. ${ }^{17}$ At $\mathrm{pH}>10$ (100\% ionization degree of the fatty acid), both G-C18:1 and G-C18:0 systems are composed by more than $\sim 95 \%$ (by number) of micelles, as estimated by the micelle/membrane ratio customized model (eq S3) used to generate the data in Figure 6 . The remaining $5 \%$ account for large objects responsible for the low- $q$ scattering signal present in Figure 2. Data in Figure 2 and Table S1 also show that GC18:0 is able to stabilize highly curved, spherical, micellar aggregates, while the presence of a saturated double bond (GC18:1) destabilizes the system enough to form slightly ellipsoidal objects. By increasing the $\mathrm{COOH} / \mathrm{COO}^{-} \mathrm{Na}^{+}$ratio, the difference between both systems becomes more pronounced as G-C18:1 continuously forms infinitely long cylindrical micelles (strong increase in aspect ratio (core) in Figure 5 and $D_{\mathrm{f}}$ approaching -1 in Figure 4), while micelles are stable and remain spherical in the G-C18:0 system (constant core aspect ratio in Figure 5). These differences indicate that the high flexibility of the hydrophobic core for G-C18:1 (room temperature, RT $>T_{M}$ of oleic acid, $13{ }^{\circ} \mathrm{C}$ ), the smaller inter$\mathrm{COO}^{-}$repulsion contribution, and the decreasing surface area per hydrophilic headgroup due to water-withdrawing effects permit the unidimensional micellar growth. On the contrary, the stiffer saturated fatty acid (RT $\ll T_{M}$ of stearic acid) does not allow the same core flexibility and spherical micelles are eventually more resistant toward the $\mathrm{COOH} / \mathrm{COO}^{-} \mathrm{Na}^{+}$ratio and hydration evolution. However, at a critical $\mathrm{COOH} /$ $\mathrm{COO}^{-} \mathrm{Na}^{+}$composition, a sharp morphological transition must take place to reduce the system energy and to take into account the new geometrical constraints. Below the respective transition $\mathrm{pH}$, each glucolipid finds itself in a membrane geometry; however, G-C18:1 forms vesicles $\left(V_{\mathrm{f}}>2\right)$, while GC18:1 infinitely grows into large sheets $\left(V_{\mathrm{f}}<1\right)$. If one relies on the model in ref 17 , two parameters play a crucial role in this system, the bending energy and line tension. The use of a 


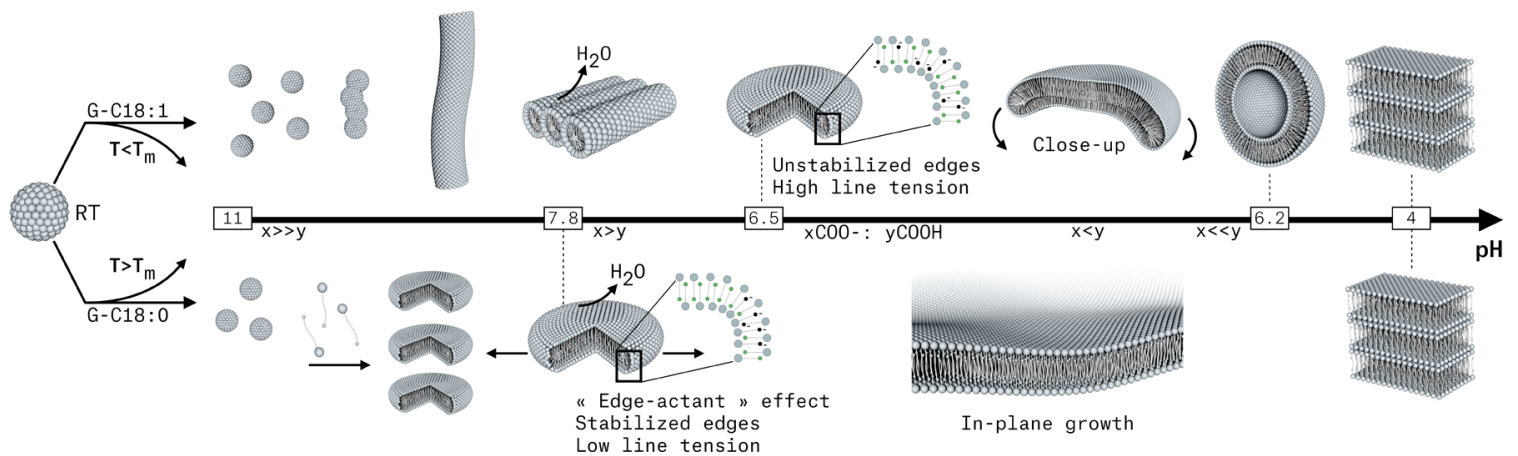

Figure 7. Scheme illustrating the $\mathrm{pH}$-driven self-assembly of G-C18:1 and G-C18:0 glucolipids in water at room temperature. $x$ and $y$ represent the fraction of $\mathrm{COO}^{-}$and $\mathrm{COOH}$ groups during $\mathrm{pH}$ change. Unless otherwise indicated, experiments have been done at room temperature (RT). The $T_{\mathrm{m}}$ for oleic acid (contained in G-C18:1) is $13{ }^{\circ} \mathrm{C}$, while the $T_{\mathrm{m}}$ for stearic acid (contained in G-C18:0) is $69{ }^{\circ} \mathrm{C}$.

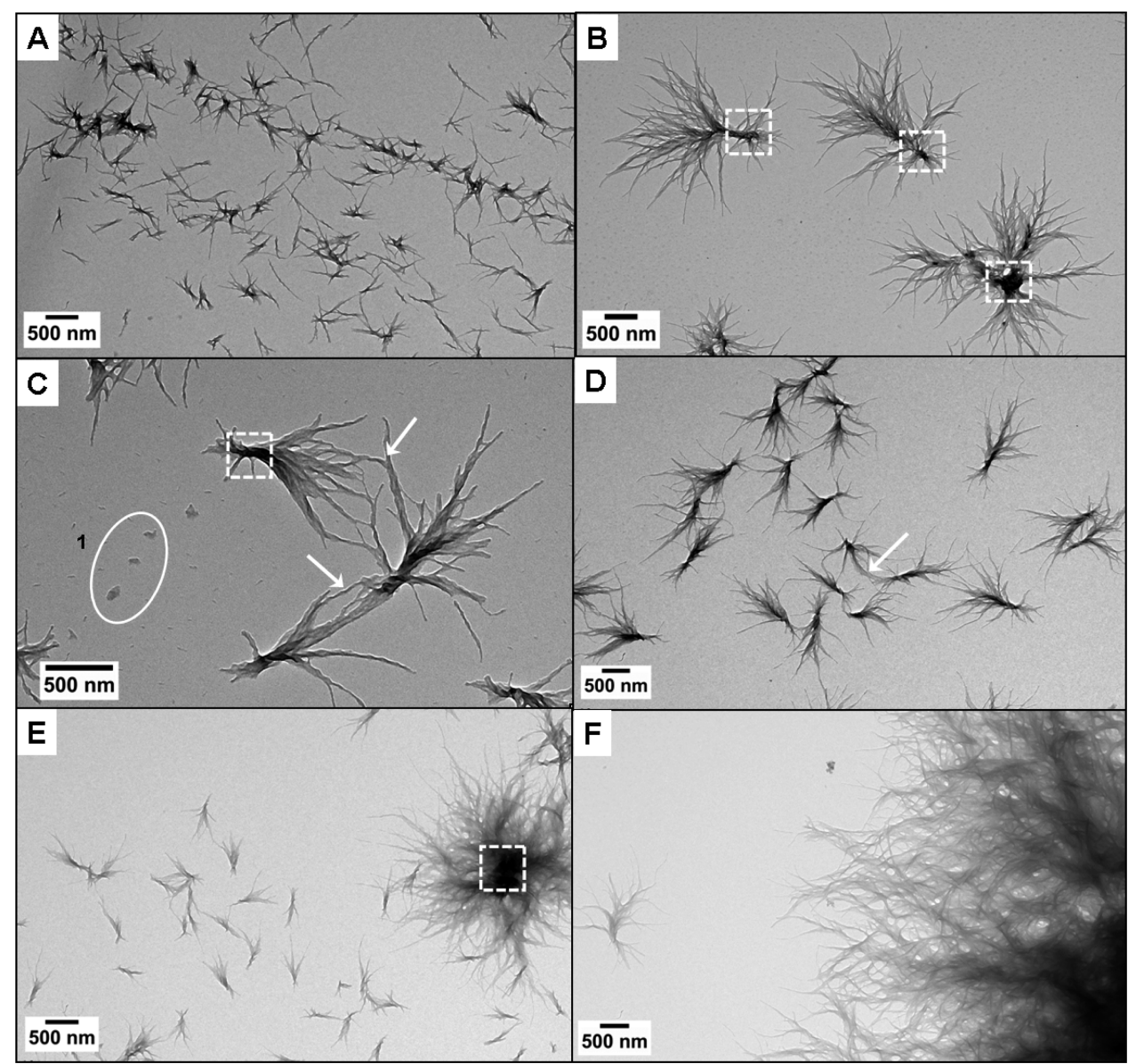

Figure 8. TEM images collected on a copper TEM grid containing a drop of SL-C18:1 sample at pH 11 (0.5 wt \%) and locally exposed for a few seconds to a $0.1 \mathrm{M} \mathrm{HCl}$ solution. The drop is immediately dried, and the grid is analyzed as such. All images come from the same grid, and they have been tried to be collected in the grid region at the opposite of the point of injection of $\mathrm{HCl}$.

saturated fatty acid (stearic acid in G-C18:0) with a high melting temperature $\left(69{ }^{\circ} \mathrm{C}\right)$ compared to room temperature seems to be responsible for the formation of a highly stable bilayer membrane, stiff and with a high bending energy, contributing to reduce $V_{\mathrm{f}}$. Meanwhile, the line tension must also be very small to account for such stable sheet structures even for "infinite" membrane radii. This is possible for an important concentration of charged species at the edges of the membrane covering an important rim area fraction. The GC18:0 system seems to better accommodate to high curvatures (at the rim) than G-C18:1, as also demonstrated by the fact that G-C18:0 forms highly stable spherical micelles, while GC18:1 forms ellipsoidal and cylindrical objects; we then make the hypothesis that the membrane edges in the G-C18:0 system are rich in $\mathrm{COO}^{-} \mathrm{Na}^{+}$, thus preventing high line tension and consequent closing of the sheet into a vesicle. On the contrary, the amount of $\mathrm{COO}^{-} \mathrm{Na}^{+}$in the G-C18:1 system is most likely diluted throughout the membrane rather than being localized at the rim, the main reason being the higher fluidity of the oleic acid tail, the $T_{M}$ of which is lower than room temperature. These mechanisms are summarized in Figure 7. 


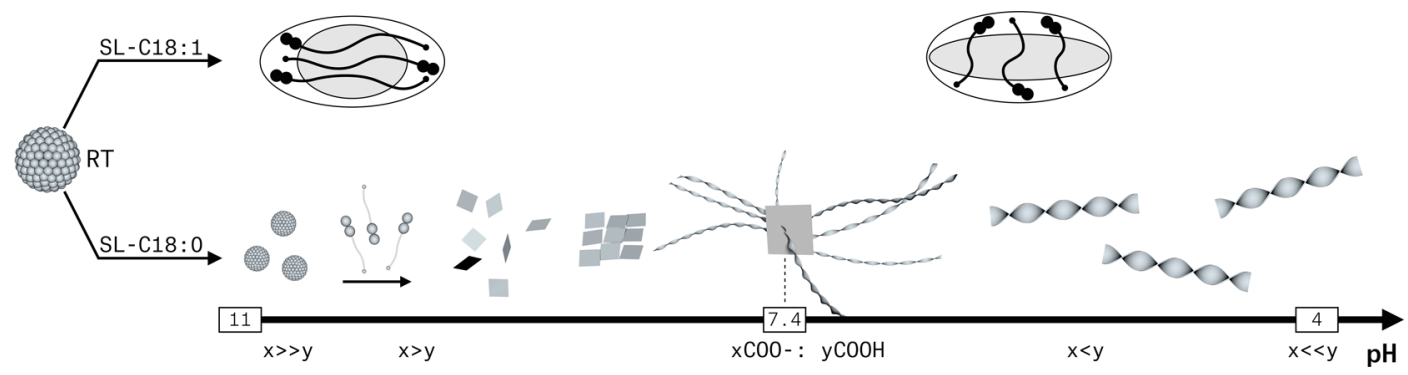

Figure 9. Scheme illustrating the $\mathrm{pH}$-driven self-assembly of SL-C18:1 and SL-C18:0 glycolipids in water at room temperature. $x$ and $y$ represent the fraction of $\mathrm{COO}^{-}$and $\mathrm{COOH}$ groups during $\mathrm{pH}$ change.

This hypothesis does not explain all of our observations. At $\mathrm{pH}$ values below 5-6, the overall content of $\mathrm{COO}^{-} \mathrm{Na}^{+}$ glucolipid species becomes very small and vesicles should form for all systems, which is not the case. To explain this phenomenon, we speculate that a critical radius exists and above which the bilayers are so large that even for high line tension, the energetic cost to bend them into a vesicle is so high that the flat membrane configuration is still preferred. We should emphasize the fact that, below $\mathrm{pH} 5$, the G-C18:0 sheets precipitate into a lamellar phase, just as found for G-C18:1. This effect seems to go in the sense of the previous hypothesis. In the G-C18:1 system, the residual $\mathrm{COO}^{-} \mathrm{Na}^{+}$are homogeneously distributed in the entire bilayer membrane, helping to stabilize the vesicle mild curvature, as observed in ref 17 for diluted bile salt systems. When all carboxylate groups are protonated, the vesicle curvature becomes so small that a lamellar phase eventually precipitates. Finally, we must recall that, if these experiments are performed at $T<13{ }^{\circ} \mathrm{C}$, G-C18:1 forms flat bilayer sheets, while, if performed at $T>69^{\circ} \mathrm{C}$, GC18:0 forms vesicles, ${ }^{39}$ thus showing the direct importance of membrane flexibility in relationship with the fluidity of the hydrophobic region. Even though this is not a direct proof, these additional data suggest that, if the mobility of the charged lipids within the membrane is affected, it is possible to control their local concentration and eventually the long-range curvature of the bilayer membrane. This was shown by other authors. Madenci et al. ${ }^{59}$ have built a model on lecithin and bile salt mixtures confirming Leng's observations and, at least from a qualitative point of view, they do show that the end-caps of bilayer disks are richer in bile salt, while lecithin is distributed in the bilayer. Other works on catanionic surfactant mixtures also imply partitioning of lipids at a molecular level, as proposed in refs $60-62$ and shown by means of contrast matching small angle neutron scattering. ${ }^{6}$

Additional Insights into the Mechanisms of Formation. Superimposing the $\mathrm{pH}$-dependent SAXS data presented in Figure 3 shows an interesting, very unique feature: practically all systems have at least one value of the wavevector $q$ which is constant throughout the variation in $\mathrm{pH}$. This invariant is commonly called the "isoscattering", or "isosbestic scattering", point. Even if a similar scattering behavior has been reported for several systems both in $\mathrm{SAXS}^{63,64}$ and $\mathrm{XRD}^{65}$ studies, for instance, in heat-induced aggregation and gelation of $\beta$ lactoglobulin and ovalbumin mixtures, ${ }^{63}$ or temperatureinduced structural modification of poly $(N$-isopropylacrylamide $)$ hydrogels, the real significance of an isoscattering point, although generally attributed to microphase separation processes, is still unclear, and in particular the meaning of the isoscattering $q$-value itself (please refer to the Supporting Information for more details). In spite of a better interpretation of isoscattering points in the literature, we apply the formation of stable "nanopockets" (synonym term to microdomain employed in ref 64) reported in the literature to the systems studied here. The SL-C18:0 system displays a narrow $q_{\text {iso }}$ interval (Figure S5) centered at $0.56 \mathrm{~nm}^{-1}(11.21 \mathrm{~nm})$, corresponding to the size of several micellar aggregates (at basic $\mathrm{pH}$ ) and, approximately, the cross section of a single ribbon. ${ }^{42,38}$ We speculate that the presence of an isoscattering point could also indicate the existence of a nucleation and growth mechanism starting from the nanopockets formed at basic $\mathrm{pH}$. This hypothesis seems to be confirmed by complementary standard TEM analysis performed on a grid on which the initial ribbon growth stages has been quenched (see the Materials and Methods for details). Figure 8A-D presents a large number of fibrillar objects of which the size does not exceed a few micrometers. Meanwhile, in Figure 8E,F, larger objects compose a dense network of entangled fibers. The entire set of images nicely demonstrates the fact that the same grid contains SL-C18:0 fibers at an early (A-D) and late (E,F) stage of formation. A closer look at Figure $8 \mathrm{~A}-\mathrm{D}$ illustrates the presence of a large number of aggregates all having a point in common: each one has a "bouquet"-like morphology, with a dense elongated center (dotted squares in Figure $8 \mathrm{~B}, \mathrm{C}$ ) and a fibrillar ramification on both extremities. The arrows in Figure 8C,D draw the attention on how these "bouquet"-like aggregates connect together via their fibrillar ramifications to eventually form a broader network (Figure 8D). Objects with a high fibrillation density in Figure 8E show that their development can also occur on a single nucleation site, which can phagocytize nearby germs. Eventually, midsized fibrillar pellets indefinitely grow by both fibrillation and aggregation into very large aggregates (Figure $8 \mathrm{E}$ ). The presence of large spherical fibrous pellets has been demonstrated before in cryo-TEM experiments collected on assynthesized SL-C18:0 sample at acidic $\mathrm{pH}^{43}$ thus indicating that the observation method preferred in this work does not influence the formation mechanism of the fibers.

At basic $\mathrm{pH}$, the SL-C18:0 is composed of micelles and, in minor amounts, of platelets. Two hypotheses can then be formulated. Clusters of micelles start to form upon lowering the $\mathrm{pH}$, becoming the nucleation point of the fibers. The core size of the nucleation point/nanopocket would remain constant, thus providing the isoscattering point in SAXS experiments. This mechanism seems to be in line with previously reported mechanisms for amyloid fibrillogenesis and in which micellar aggregates are believed to be both the nucleation point and reservoir. ${ }^{66-68}$ However, one can wonder which is the driving force for micelles to spontaneously aggregate into nanopockets. To overcome this question, we formulate a second hypothesis according to which the platelets constitute the seed from which 
the fibers nucleate and develop. Against this hypothesis, one should highlight the fact that the platelet aggregates disappear with lowering $\mathrm{pH}$, as shown by the diminishing low- $q$ signal between $\mathrm{pH} 12$ and $\mathrm{pH} 10$ on the SL-C18:0 sample in Figure 4. However, that signal is related to large (several micrometers) platelet aggregates and not to a single platelet, as shown in ref 38. On the contrary, other arguments support the second hypothesis. Similarities are found in the formation of twisted/ helical ribbons from gemini surfactant systems, which form platelets in the presence of a racemate mixture of D-, L-tartrate counterions but form ribbons in the enantiomeric excess of one or the other. ${ }^{13}$ More generally, recent studies on the fibrillation mechanism of silk fibers (using SAXS/WAXS/Raman), ${ }^{69}$ peptide nanotubes (using SAXS, cryo electron tomograghy, and cryo TEM), ${ }^{16}$ and amyloid fibers (Monte Carlo simulation $)^{70}$ all seem to exclude the nucleation process from micelles but rather converge toward an aggregation/conversion (for silk and amyloid) or oriented attachment (peptide nanotubes) set of mechanisms. We conclude then that micelles, present at basic $\mathrm{pH}$, serve as a reservoir of matter but not as nucleation points. This is shown in the scheme in Figure 9, which also reports the micellar structural evolution for the SLC18:1 sophorolipids for a matter of comparison.

\section{CONCLUSION}

This work unravels the pH-dependent self-assembly mechanism of a set of four glycolipids bearing a single glucose and a sophorose headgroup and oleic, or stearic, acid as lipid backbone. These structurally related compounds are obtained directly, or indirectly, from the fermentation process of the yeast Starmerella bombicola. All four compounds form spherical, or ellipsoidal, micelles under basic $\mathrm{pH}$ conditions due to the strong electrostatic repulsion between the $\mathrm{COO}^{-}$groups. The structures of the micelles mainly vary in terms of the glycolipid distribution within the micelle itself. Upon lowering the $\mathrm{pH}$ of the solution, each glycolipid follows its own pathway toward different structures. We have used $\mathrm{pH}$-resolved in situ SAXS using synchrotron radiation to follow the morphological changes; the SAXS data have been thoroughly analyzed using both a model-independent and a model-dependent approach. The $\mathrm{pH}$-dependent analysis of the slope in the low- $q$ region informs on the nature of the large aggregates, while the evolution of the minimum of the form factor oscillation, $q_{\min }$, informs on the morphology changes. Then, we have modeled the micellar region using a core-shell ellipsoid of revolution form factor using an inhomogeneous shell, necessary to fit part of the data. Finally, when appropriate, we have used a coreshell bicelle model to fit the membrane morphologies (vesicle, bilayer).

We find the following:

- G-C18:1 system: Micelles are ellipsoids at basic $\mathrm{pH}$, and they elongate and merge into cylindrical and wormlike objects, which in turn fuse into large disks in the narrow $\mathrm{pH}$ region between 7.5 and 6.5., where glucolipids are interdigitated. This is promoted by (1) the fluid hydrophobic region $\left(\mathrm{RT}>T_{\mathrm{m}}\right),(2)$ the intrinsic value of the packing parameter ( 0.72$)$, and (3) the dehydration process. (4) High line tension at the disk extremities promoting vesicle formation at $\mathrm{pH}<6.2$. (5) Formation of a lamellar phase below $\mathrm{pH} 4$, presumably when all $\mathrm{COO}^{-}$turn into $\mathrm{COOH}$.
- G-C18:0 system: Micelles are spherical and very stable in a broad $\mathrm{pH}$ region. They coexist with a small fraction (about 5\%) of large flat objects which experience a sudden increase in size below the transition $\mathrm{pH} 8$, where glucolipids are interdigitated. Micelles either fuse in the preformed membranes or dissolve to provide matter for the bilayer growth. In all cases, they seem to act as a reservoir. The bilayer does not bend into vesicles, but it rather grows infinitely into extended bilayers, possibly because of the edge-stabilizing effect of the edge-actants, composed of the residual glucolipid bearing a $\mathrm{COO}^{-}$ group. A lamellar phase eventually precipitates presumably when all $\mathrm{COO}^{-}$groups are replaced by $\mathrm{COOH}$.

- SL-C18:1 system: Micelles are ellipsoids with a given sophorolipid distribution inside. Upon lowering the $\mathrm{pH}$, the chain fluidity, the strong hydration of sophorose, and the packing parameter (0.36) drive the formation of elongated ellipsoids, although with a different distribution of sophorolipids inside, thus forming coffee-beanlike objects. ${ }^{41}$ If platelets are also reported to coexist with micelles at basic $\mathrm{pH}^{43}$ they disassemble with lowering the $\mathrm{pH}$ and serve as a source of matter for micelle rearrangement.

- SL-C18:0 system: Micelles are spherical stable objects until the transition $\mathrm{pH}$. The formation of the ribbons occurs at $\mathrm{pH} 7.4$ from, we believe, preformed nanoscale platelets serving as nucleation points. ${ }^{43}$ Micelles serve as a source of matter for ribbon formation. The driving force for ribbon formation is still unclear, but it could be related to the bulky sophorose perturbation effect during the formation of a flat bilayer.

In the end, this work is a didactical illustration of the complexity of the self-assembly process of a stimuli-responsive amphiphile and during which many concomitant parameters play a key role at different stages of the process. We believe that this work should provide a step forward in the prediction of such complex systems.

\section{ASSOCIATED CONTENT}

\section{S Supporting Information}

The Supporting Information is available free of charge on the ACS Publications website at DOI: 10.1021/acs.langmuir.6b02337.

(Figure S1) HPLC-ELSD chromatogram of the SLC18:1 compound and description of the strategy to fit the SAXS data; (Figure S2) the general overview of the treatment performed on a typical SAXS spectrum; (Figure S3) the contour plots of the $\mathrm{pH}$-resolved SAXS experiments for all glycolipids; (Figure S4) the $\mathrm{pH}$ dependent evolution of the micelle and membrane bilayer structural parameters for G-C18:1 and G-C18:0 samples obtained after fitting the SAXS data; (Figure S5) the isoscattering point for SL-C18:0; (Table S1) the parameters used to fit the SAXS data presented in Figure 2; also the hypotheses for applying Leng's model to the G-C18:1 and G-C18:0 systems (PDF)

\section{AUTHOR INFORMATION}

\section{Corresponding Author}

*E-mail: niki.baccile@upmc.fr.

\section{Notes}

The authors declare no competing financial interest. 


\section{ACKNOWLEDGMENTS}

SAXS experiments were performed on beamline ID02 at the European Synchrotron Radiation Facility (ESRF), Grenoble, France. The research leading to these results has received funding from the European Community's Seventh Framework Programme (FP7/2007-2013) under Grant Agreement No. Biosurfing/289219. This work benefits from SasView software, developed by the DANSE project under NSF award DMR0520547. David Benque (Protoplot, www.protoplot.com) is acknowledged for the graphic design images.

\section{REFERENCES}

(1) Felber, E.; Dufresne, M.-H.; Leroux, J.-C. pH-sensitive vesicles, polymeric micelles, and nanospheres prepared with polycarboxylates. Adv. Drug Delivery Rev. 2012, 64, 979-992.

(2) Brown, P.; Butts, C. P.; Eastoe, J. Stimuli-responsive surfactants. Soft Matter 2013, 9, 2365-2374.

(3) Li, X.; Yang, Y.; Eastoe, J.; Dong, J. Rich Self-Assembly Behavior from a Simple Amphiphile. ChemPhysChem 2010, 11, 3074-3077.

(4) Du, J.; Tang, Y.; Lewis, A. L.; Armes, S. P. pH-Sensitive Vesicles Based on a Biocompatible Zwitterionic Diblock Copolymer. J. Am. Chem. Soc. 2005, 127, 17982-17983.

(5) Morigaki, K.; Walde, P. Fatty acid vesicles. Curr. Opin. Colloid Interface Sci. 2007, 12, 75-80.

(6) Israelachvili, J. N.; Mitchell, D. J.; Ninham, B. W. Theory of selfassembly of hydrocarbon amphiphiles into micelles and bilayers. $J$. Chem. Soc., Faraday Trans. 2 1976, 72, 1525-1568.

(7) Nagarajan, R.; Ruckenstein, E. Theory of Surfactant Self -Assembly: A Predictive Molecular Thermodynamic Approach. Langmuir 1991, 7, 2934-2969.

(8) Berr, S. S.; Caponetti, E.; Johnson, J. S., Jr.; Jones, R. R. M.; Magid, L. J. Small-angle neutron scattering from hexadecyltrimethylammonium bromide micelles in aqueous solutions. J. Phys. Chem. 1986, 90, 5766-5770.

(9) Alexandridis, P.; Athanassiou, V.; Fukuda, S.; Hatton, T. A. Surface Activity of Poly(ethyleneoxide)-block-Poly(propylene oxide)block-Poly(ethyleneoxide) Copolymers. Langmuir 1994, 10, 26042612

(10) Klijn, J. E.; Stuart, M. C. A.; Scarzello, M.; Wagenaar, A.; Engberts, J. B. F. N. pH-Dependent Phase Behavior of CarbohydrateBased Gemini Surfactants. The Effects of Carbohydrate Stereochemistry, Head Group Hydrophilicity, and Nature of the Spacer. J. Phys. Chem. B 2007, 111, 5204-5211.

(11) Nagarajan, R. Molecular Packing Parameter and Surfactant SelfAssembly: The Neglected Role of the Surfactant Tail. Langmuir 2002, $18,31-38$

(12) Zana, R. Gemini (dimeric) surfactants. Curr. Opin. Colloid Interface Sci. 1996, 1, 566-571.

(13) Oda, R.; Huc, I.; Schmutz, M.; Candau, S. J.; MacKintosh, F. C. Tuning bilayer twist using chiral counterions. Nature 1999, 399, 566569.

(14) Rand, R. P.; Fuller, N. L.; Gruner, S. M.; Parsegian, V. A. Membrane Curvature, Lipid Segregation, and Structural Transitions for Phospholipids under Dual-Solvent Stress? Biochemistry 1990, 29, $76-87$.

(15) Guida, V. Thermodynamics and kinetics of vesicles formation processes. Adv. Colloid Interface Sci. 2010, 161, 77-88.

(16) Cenker, C. C.; Bomans, P. H. H.; Friedrich, H.; Dedeoglu, B.; Aviyente, V.; Olsson, U.; Sommerdijk, N. A. J. M.; Bucak, S. Peptide nanotube formation: a crystal growth process. Soft Matter 2012, 8, 7463.

(17) Leng, J.; Egelhaaf, S. U.; Cates, M. E. Kinetics of the Micelle-toVesicle Transition: Aqueous Lecithin-Bile Salt Mixtures. Biophys. J. 2003, 85, 1624-1646.

(18) Genc, R.; Ortiz, M.; O'Sullivan, C. K. Curvature-Tuned Preparation of Nanoliposomes. Langmuir 2009, 25, 12604-12613.
(19) Bryskhe, K.; Bulut, S.; Olsson, U. Vesicle Formation from Temperature Jumps in a Nonionic Surfactant System. J. Phys. Chem. B 2005, 109, 9265-9274.

(20) Corti, M.; Cantù, L.; Brocca, P.; Del Favero, E. Self-assembly in glycolipids. Curr. Opin. Colloid Interface Sci. 2007, 12, 148-154.

(21) John, G.; Masuda, M.; Okada, Y.; Yase, K.; Shimizu, T. Nanotube formation from Renewable Resources via Coiled Nanotubes. Adv. Mater. 2001, 13, 715-718.

(22) Garamus, V. M.; Milkereit, G.; Gerber, S.; Vill, V. Micellar structure of a sugar based bolaamphiphile in pure solution and destabilizing effects in mixtures of glycolipids. Chem. Phys. Lett. 2004, 392, 105-109.

(23) Desai, J. D.; Banat, I. M. Microbial production of surfactants and their commercial potential. Microbiol. Mol. Biol. Rev. 1997, 61, 47-64.

(24) Shimizu, T. Molecular Self-Assembly into One-Dimensional Nanotube Architectures and Exploitation of Their Functions. Bull. Chem. Soc. Jpn. 2008, 81, 1554-1566.

(25) Jung, J. H.; Shinkai, S.; Shimizu, T. Spectral Characterization of Self-Assemblies of Aldopyranoside Amphiphilic Gelators: What is the Essential Structural Difference between Simple Amphiphiles and Bolaamphiphiles. Chem. - Eur. J. 2002, 8, 2684-2690.

(26) Kitamoto, D.; Morita, T.; Fukuoka, T.; Konishi, M.; Imura, T. Self-assembling properties of glycolipid biosurfactants and their potential applications. Curr. Opin. Colloid Interface Sci. 2009, 14, 315-328.

(27) Fuhrhop, J.-H.; Wang, T. Bolaamphiphiles. Chem. Rev. 2004, 104, 2901-2937.

(28) Kamiya, S.; Minamikawa, H.; Jung, J. H.; Yang, B.; Masuda, M.; Shimizu, T. Molecular Structure of Glucopyranosylamide Lipid and Nanotube Morphology. Langmuir 2005, 21, 743-750.

(29) Axford, J. The impact of glycobiology on medicine. Trends Immunol. 2001, 22, 237.

(30) Dwek, R. A. Glycobiology: Toward Understanding the Function of Sugars. Chem. Rev. 1996, 96, 683-720.

(31) Kameta, N.; Minamikawa, H.; Masuda, M. Supramolecular organic nanotubes: how to utilize the inner nanospace and the outer space. Soft Matter 2011, 7, 4539.

(32) Marchant, R.; Banat, I. Biosurfactants: a sustainable replacement for chemical surfactants? Biotechnol. Lett. 2012, 34, 1597-1605.

(33) Marchant, R.; Banat, I. Microbial biosurfactants: challenges and opportunities for future exploitation. Trends Biotechnol. 2012, 30, 558565 .

(34) Van Bogaert, I. N. A.; Saerens, K.; De Muynck, C.; Develter, D.; Soetaert, W.; Vandamme, E. J. Microbial production and application of sophorolipids. Appl. Microbiol. Biotechnol. 2007, 76, 23-34.

(35) Roelants, S. L.; Ciesielska, K.; De Maeseneire, S. L.; Moens, H.; Everaert, B.; Verweire, S.; Denon, Q.; Vanlerberghe, B.; Van Bogaert, I. N. A.; Van der Meeren, P.; Devreese, B.; Soetaert, W. Towards the industrialization of new biosurfactants: Biotechnological opportunities for the lactone esterase gene from Starmerella bombicola. Biotechnol. Bioeng. 2016, 113, 550-559.

(36) Masuda, M.; Yozac, K.; Shimizu, T. Polymorphism of monolayer lipid membrane structures made from unsymmetrical bolaamphiphiles. Carbohydr. Res. 2005, 340, 2502-2509.

(37) Frankel, D. A.; O’Brien, D. F. Supramolecular Assemblies of Diacetylenic Aldonamides. J. Am. Chem. Soc. 1994, 116, 10057-10069.

(38) Cuvier, A.-S.; Babonneau, F.; Berton, J.; Stevens, C. V.; Fadda, G. C.; Péhau-Arnaudet, G.; Le Griel, P.; Prévost, S.; Perez, J.; Baccile, $\mathrm{N}$. Nanoscale platelet formation by monounsaturated and saturated sophorolipids under basic $\mathrm{pH}$ conditions. Chem. - Eur. J. 2015, 21, 19265-19277.

(39) Baccile, N.; Selmane, M.; Le Griel, P.; Prévost, S.; Perez, J.; Stevens, C. V.; Delbeke, E.; Zibek, S.; Guenther, M.; Soetaert, W.; Van Bogaert, I. N. A.; Roelants, S. pH-driven self-assembly of acidic microbial glycolipids. Langmuir 2016, 32, 6343.

(40) Baccile, N.; Babonneau, F.; Jestin, J.; Pehau-Arnaudet, G.; Van Bogaert, I. Unusual, pH-induced, self-assembly of sophorolipid biosurfactants. ACS Nano 2012, 6, 4763-4776. 
(41) Manet, S.; Cuvier, A.-S.; Valotteau, C.; Fadda, G. C.; Perez, J.; Karakas, E.; Abel, S.; Baccile, N. Structure of Bolaamphiphile Sophorolipid Micelles Characterized with SAXS, SANS, and MD Simulations. J. Phys. Chem. B 2015, 119, 13113-13133.

(42) Cuvier, A.-S.; Berton, J.; Stevens, C. V.; Fadda, G. C.; Babonneau, F.; Van Bogaert, I. N. A.; Soetaert, W.; Péhau-Arnaudet, G.; Baccile, N. pH-triggered formation of nanoribbons from yeast derived glycolipid biosurfactants. Soft Matter 2014, 10, 3950-3959.

(43) Cuvier, A.-S.; Babonneau, F.; Berton, J.; Stevens, C. V.; Fadda, G. C.; Genois, I.; Le Griel, P.; Péhau-Arnaudet, G.; Baccile, N. Synthesis of Uniform, Monodisperse, Sophorolipid Twisted Ribbons. Chem. - Asian J. 2015, 10, 2419-2426.

(44) Ballauff, M.; Jusufi, A. Anomalous small-angle X-ray scattering: analyzing correlations and fluctuations in polyelectrolytes. Colloid Polym. Sci. 2006, 284, 1303-1311.

(45) Jusufi, A. Fluctuation effects and monomer-counterion correlations in starlike polyelectrolyte systems. J. Chem. Phys. 2006, $124,044908$.

(46) Nagarajan, R. Self-assembly of bola amphiphiles. Chem. Eng. Commun. 1987, 55, 251-273.

(47) Glatter, O.; Kratky, O. Small Angle X-ray Scattering; Academic Press: London, 1982.

(48) Teixeira, J. Small-Angle Scattering by Fractal Systems. J. Appl. Crystallogr. 1988, 21, 781-785.

(49) Max, J.-J.; Chapados, C. Infrared Spectroscopy of Aqueous Carboxylic Acids: Comparison between Different Acids and Their Salts. J. Phys. Chem. A 2004, 108, 3324-3337.

(50) Tait, M. J.; Suggett, A.; Franks, F.; Ablett, S.; Quickenden, P. A. Hydration of monosaccharides: A study by dielectric and nuclear magnetic relaxation. J. Solution Chem. 1972, 1, 131-151.

(51) Miyajima, K.; Sawada, M.; Nakagaki, M. Studies on Aqueous Solutions of Saccharides. II. Viscosity B-Coefficients, Apparent Molar Volumes, and Activity Coefficients of D-Glucose, Maltose, and Maltotriose in Aqueous Solutions. Bull. Chem. Soc. Jpn. 1983, 56, 1954-1957.

(52) Li, X.-J.; Schick, M. Theory of Tunable pH-Sensitive Vesicles of Anionic and Cationic Lipids or Anionic and Neutral Lipids. Biophys. J. 2001, 80, 1703-1711.

(53) Hafez, I. M.; Cullis, P. R. Cholesteryl hemisuccinate exhibits $\mathrm{pH}$ sensitive polymorphic phase behavior. Biochim. Biophys. Acta, Biomembr. 2000, 1463, 107-114.

(54) Hafez, I. M.; Ansell, S.; Cullis, P. R. Tunable pH sensitive liposomes composed of mixtures of cationic and anionic lipids. Biophys. J. 2000, 79, 1438-1446.

(55) LeNeveu, D. M.; Rand, R. P.; Parsegian, V. A.; Gingell, D. Measurement and modification of forces between lecithin bilayers. Biophys. J. 1977, 18, 209-230.

(56) Rand, R. P.; Fuller, N.; Parsegian, V. A.; Rau, D. C. Variation in Hydration Forces between Neutral Phospholipid Bilayers: Evidence for Hydration Attraction. Biochemistry 1988, 27, 7711-7722.

(57) Di Gregorio, G. M.; Mariani, P. Rigidity and spontaneous curvature of lipidic monolayers in the presence of trehalose: a measurement in the DOPE inverted hexagonal phase. Eur. Biophys. J. 2005, 34, 67-81.

(58) Fromherz, P. Lipid-vesicle structure: size control by edge-active, agents. Chem. Phys. Lett. 1983, 94, 259-266.

(59) Madenci, D.; Salonen, A.; Schurtenberger, P.; Pedersen, J. S.; Egelhaaf, S. U. Simple model for the growth behaviour of mixed lecithin-bile salt micelles. Phys. Chem. Chem. Phys. 2011, 13, 31713178.

(60) Zemb, Th.; Dubois, M.; Deme, B.; Gulik-Krzywicki, Th. Selfassembly of flat nanodiscs in salt-free catanionic surfactant solutions. Science 1999, 283, 816.

(61) Carriere, D.; Belloni, L.; Deme, B.; Dubois, M.; Vautrin, C.; Meister, A.; Zemb, Th. In-plane distribution in mixtures of cationic and anionic surfactants. Soft Matter 2009, 5, 4983.

(62) Manohar, C.; Narayanan, J. Edge-edge attractions in surfactant nanodiscs: Implications. Chem. Phys. Lett. 2011, 517, 176-179.
(63) Nicolai, T.; Pouzot, M.; Durand, D.; Weijers, M.; Visschers, R $\mathrm{W}$. Iso-scattering points during heat-induced aggregation and gelation of globular proteins indicating micro-phase separation. Europhys. Lett. 2006, 73, 299-305.

(64) Chalal, M.; Ehrburger-Dolle, F.; Morfin, I.; Bley, F.; Aguilar de Armas, M.-R.; Lopez Donaire, M.-L.; San Roman, J.; Bolgen, N.; Piskin, E.; Ziane, O.; Casalegno, R. SAXS Investigation of the Effect of Temperature on the Multiscale Structure of a Macroporous Poly $(\mathrm{N}$ isopropylacrylamide) Gel. Macromolecules 2010, 43, 2009-2017.

(65) Johnson, R. W. Diffraction Isosbestic Points And Structural Systematics In The $\mathrm{Si}_{\mathrm{x}}, \mathrm{Se}_{1-\mathrm{x}}$ Glass System. J. Non-Cryst. Solids 1986, 88, 366-380.

(66) Walsh, D. M.; Lomakin, A.; Benedek, G. B.; Condron, M. M.; Teplow, D. B. Amyloid $\beta$-Protein Fibrillogenesis Detection of A Protofibrillar Intermediate. J. Biol. Chem. 1997, 272, 22364-22372.

(67) Lomakin, A.; Chung, D. S.; Benedek, G. B.; Kirschner, D. A.; Teplow, D. B. On the nucleation and growth of amyloid, 8-protein fibrils: Detection of nuclei and quantitation of rate constants. Proc. Natl. Acad. Sci. U. S. A. 1996, 93, 1125-1129.

(68) Sabate, R.; Estelrich, J. Evidence of the Existence of Micelles in the Fibrillogenesis of â-Amyloid Peptide. J. Phys. Chem. B 2005, 109, 11027-11032.

(69) Martel, A.; Burghammer, M.; Davies, R. J.; Di Cola, E.; Vendrely, C.; Riekel, C. Silk Fiber Assembly Studied by Synchrotron Radiation SAXS/WAXS and Raman Spectroscopy. J. Am. Chem. Soc. 2008, 130, 17070-17074.

(70) Auer, S.; Dobson, C. M.; Vendruscolo, M. Characterization of the nucleation barriers for protein aggregation and amyloid formation. HFSP J. 2007, 1, 137-146. 